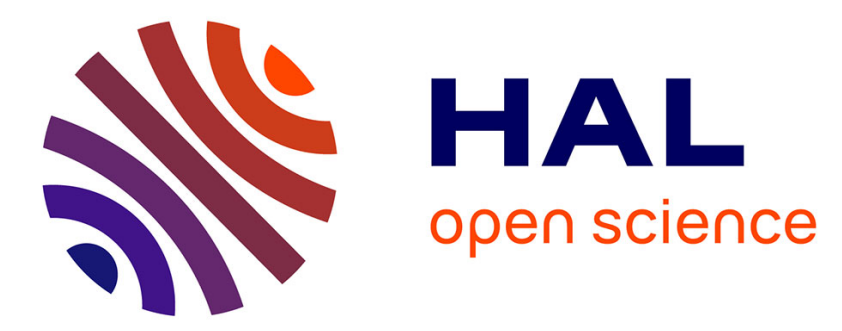

\title{
Prognostic value and time course evolution of left ventricular Global Longitudinal Strain in septic shock: an exploratory prospective study \\ Florian Bazalgette
}

\section{- To cite this version:}

Florian Bazalgette. Prognostic value and time course evolution of left ventricular Global Longitudinal Strain in septic shock: an exploratory prospective study. Human health and pathology. 2018. dumas02952342

\section{HAL Id: dumas-02952342 https://dumas.ccsd.cnrs.fr/dumas-02952342}

Submitted on 29 Sep 2020

HAL is a multi-disciplinary open access archive for the deposit and dissemination of scientific research documents, whether they are published or not. The documents may come from teaching and research institutions in France or abroad, or from public or private research centers.
L'archive ouverte pluridisciplinaire HAL, est destinée au dépôt et à la diffusion de documents scientifiques de niveau recherche, publiés ou non, émanant des établissements d'enseignement et de recherche français ou étrangers, des laboratoires publics ou privés.

\section{(1)(1) $\$(0)$}

Distributed under a Creative Commons Attribution - NonCommercial - ShareAlikel 4.0 


\section{UNIVERSITE DE MONTPELLIER \\ FACULTE DE MEDECINE MONTPELLIER-NIMES}

\section{Thèse}

Présentée et soutenue publiquement

Le 5 octobre 2018

Par

Florian BAZALGETTE

\section{Pour obtenir le titre de DOCTEUR EN MÉDECINE}

\section{Titre}

Valeur pronostique et évolution temporelle du strain global longitudinal du ventricule gauche au cours du choc septique, résultats d'une étude prospective

Prognostic value and time course evolution of left ventricular Global Longitudinal Strain in septic shock: An exploratory prospective study

\section{Directeur de thèse : Dr Claire ROGER}

Président :

Professeur Jean Yves LEFRANT

Professeur des Universités - Praticien Hospitalier

Assesseurs :

Professeur Pascal COLSON

Professeur des Universités - Praticien Hospitalier

Professeur Guillaume CAYLA

Professeur des Universités - Praticien Hospitalier

Docteur Claire ROGER

Praticien Hospitalier

Membre invité :

Professeur Laurent MULLER

Professeur Associé - Praticien Hospitalier 


\section{UNIVERSITE DE MONTPELLIER \\ FACULTE DE MEDECINE MONTPELLIER-NIMES}

\section{Thèse}

Présentée et soutenue publiquement

Le 5 octobre 2018

Par

Florian BAZALGETTE

\section{Pour obtenir le titre de DOCTEUR EN MÉDECINE}

\section{Titre}

Valeur pronostique et évolution temporelle du strain global longitudinal du ventricule gauche au cours du choc septique, résultats d'une étude prospective

Prognostic value and time course evolution of left ventricular Global Longitudinal Strain in septic shock: An exploratory prospective study

\section{Directeur de thèse : Dr Claire ROGER}

Président :

Professeur Jean Yves LEFRANT

Professeur des Universités - Praticien Hospitalier

Assesseurs :

Professeur Pascal COLSON

Professeur des Universités - Praticien Hospitalier

Professeur Guillaume CAYLA

Professeur des Universités - Praticien Hospitalier

Docteur Claire ROGER

Praticien Hospitalier

Membre invité :

Professeur Laurent MULLER

Professeur Associé - Praticien Hospitalier 


\section{ANNEE UNIVERSITAIRE 2017 - 2018}

\section{PERSONNEL ENSEIGNANT}

Professeurs Honoraires

ALLIEU Yves

ALRIC Robert

ARNAUD Bernard

ASTRUC Jacques

AUSSILLOUX Charles

AVEROUS Michel

AYRAL Guy

BAILLAT Xavier

BALDET Pierre

BALDY-MOULINIER Michel

BALMES Jean-Louis

BALMES Pierre

BANSARD Nicole

BAYLET René

BILLIARD Michel

BLARD Jean-Marie

BLAYAC Jean Pierre

BLOTMAN Francis

BONNEL François

BOUDET Charles

BOURGEOIS Jean-Marie

BRUEL Jean Michel

BUREAU Jean-Paul

BRUNEL Michel

CALLIS Albert
DIMEGLIO Alain

DU CAILAR Jacques

DUBOIS Jean Bernard

DUMAS Robert

DUMAZER Romain

ECHENNE Bernard

FABRE Serge

FREREBEAU Philippe

GALIFER René Benoît

GODLEWSKI Guilhem

GRASSET Daniel

GROLLEAU-RAOUX Robert

GUILHOU Jean-Jacques

HERTAULT Jean

HUMEAU Claude

JAFFIOL Claude

JANBON Charles

JANBON François

JARRY Daniel

JOYEUX Henri

LAFFARGUE François

LALLEMANT Jean Gabriel

LAMARQUE Jean-Louis

LAPEYRIE Henri

LESBROS Daniel
MEYNADIER Jean

MICHEL François-Bernard

MICHEL Henri

MION Charles

MION Henri

MIRO Luis

NAVARRO Maurice

NAVRATIL Henri

OTHONIEL Jacques

PAGES Michel

PEGURET Claude

POUGET Régis

PUECH Paul

PUJOL Henri

PUJOL Rémy

RABISCHONG Pierre

RAMUZ Michel

RIEU Daniel

RIOUX Jean-Antoine

ROCHEFORT Henri

ROUANET DE VIGNE LAVIT Jean Pierre

SAINT AUBERT Bernard

SANCHO-GARNIER Hélène

SANY Jacques

SENAC Jean-Paul 
CANAUD Bernard

CASTELNAU Didier

CHAPTAL Paul-André

CIURANA Albert-Jean

CLOT Jacques

D'ATHIS Françoise

DEMAILLE Jacques

DESCOMPS Bernard
LOPEZ François Michel

LORIOT Jean

LOUBATIERES Marie

Madeleine

MAGNAN DE BORNIER

Bernard

MARY Henri

MATHIEU-DAUDE Pierre
SERRE Arlette

SIMON Lucien

SOLASSOL Claude

THEVENET André

VIDAL Jacques

VISIER Jean Pierre

Professeurs Emérites ARTUS Jean-Claude

BLANC François

BOULENGER Jean-Philippe

BOURREL Gérard

BRINGER Jacques

CLAUSTRES Mireille

DAURES Jean-Pierre

DAUZAT Michel

DEDET Jean-Pierre

ELEDJAM Jean-Jacques

GUERRIER Bernard

JOURDAN Jacques

MAURY Michèle

MILLAT Bertrand

MARES Pierre

MONNIER Louis

PRAT Dominique

PRATLONG Francine

PREFAUT Christian

PUJOL Rémy

ROSSI Michel

SULTAN Charles

TOUCHON Jacques

VOISIN Michel

ZANCA Michel 


\section{Professeurs des Universités - Praticiens Hospitaliers}

PU-PH de classe exceptionnelle

ALBAT Bernard - Chirurgie thoracique et cardiovasculaire

ALRIC Pierre - Chirurgie vasculaire ; médecine vasculaire (option chirurgie vasculaire)

BACCINO Eric - Médecine légale et droit de la santé

BASTIEN Patrick - Parasitologie et mycologie

BONAFE Alain - Radiologie et imagerie médicale

CAPDEVILA Xavier - Anesthésiologie-réanimation

COMBE Bernard - Rhumatologie

COSTA Pierre - Urologie

COTTALORDA Jérôme - Chirurgie infantile

COUBES Philippe - Neurochirurgie

CRAMPETTE Louis - Oto-rhino-laryngologie

CRISTOL Jean Paul - Biochimie et biologie moléculaire

DAVY Jean Marc - Cardiologie

DE LA COUSSAYE Jean Emmanuel - Anesthésiologie-réanimation

DELAPORTE Eric - Maladies infectieuses ; maladies tropicales

DE WAZIERES Benoît - Médecine interne ; gériatrie et biologie du vieillissement, médecine générale, addictologie

DOMERGUE Jacques - Chirurgie générale

DUFFAU Hugues - Neurochirurgie

DUJOLS Pierre - Biostatistiques, informatique médicale et technologies de la communication

ELIAOU Jean François - Immunologie

FABRE Jean Michel - Chirurgie générale

GUILLOT Bernard - Dermato-vénéréologie

HAMAMAH Samir-Biologie et Médecine du développement et de la reproduction ; gynécologie médicale HEDON Bernard-Gynécologie-obstétrique ; gynécologie médicale

HERISSON Christian-Médecine physique et de réadaptation

JABER Samir-Anesthésiologie-réanimation

JEANDEL Claude-Médecine interne ; gériatrie et biologie du vieillissement, médecine générale, addictologie

JONQUET Olivier-Réanimation ; médecine d'urgence

JORGENSEN Christian-Thérapeutique ; médecine d'urgence ; addictologie

KOTZKI Pierre Olivier-Biophysique et médecine nucléaire 
LANDAIS Paul-Epidémiologie, Economie de la santé et Prévention

LARREY Dominique-Gastroentérologie ; hépatologie ; addictologie

LEFRANT Jean-Yves-Anesthésiologie-réanimation

LE QUELLEC Alain-Médecine interne ; gériatrie et biologie du vieillissement, médecine générale, addictologie

MARTY-ANE Charles - Chirurgie thoracique et cardiovasculaire

MAUDELONDE Thierry - Biologie cellulaire

MERCIER Jacques - Physiologie

MESSNER Patrick - Cardiologie

MOURAD Georges-Néphrologie

PELISSIER Jacques-Médecine physique et de réadaptation

RENARD Eric-Endocrinologie, diabète et maladies métaboliques ; gynécologie médicale

REYNES Jacques-Maladies infectieuses, maladies tropicales

RIBSTEIN Jean-Médecine interne ; gériatrie et biologie du vieillissement, médecine générale, addictologie

RIPART Jacques-Anesthésiologie-réanimation

ROUANET Philippe-Cancérologie ; radiothérapie

SCHVED Jean François-Hématologie; Transfusion

TAOUREL Patrice-Radiologie et imagerie médicale

UZIEL Alain -Oto-rhino-laryngologie

VANDE PERRE Philippe-Bactériologie-virologie ; hygiène hospitalière

YCHOU Marc-Cancérologie ; radiothérapie

\section{PU-PH de $1^{\text {re }}$ classe}

AGUILAR MARTINEZ Patricia-Hématologie ; transfusion

AVIGNON Antoine-Nutrition

AZRIA David -Cancérologie ; radiothérapie

BAGHDADLI Amaria-Pédopsychiatrie ; addictologie

BEREGI Jean-Paul-Radiologie et imagerie médicale

BLAIN Hubert-Médecine interne ; gériatrie et biologie du vieillissement, médecine générale, addictologie

BLANC Pierre-Gastroentérologie ; hépatologie ; addictologie

BORIE Frédéric-Chirurgie digestive

BOULOT Pierre-Gynécologie-obstétrique ; gynécologie médicale

CAMBONIE Gilles -Pédiatrie

CAMU William-Neurologie

CANOVAS François-Anatomie 
CARTRON Guillaume-Hématologie ; transfusion

CHAMMAS Michel-Chirurgie orthopédique et traumatologique

COLSON Pascal-Anesthésiologie-réanimation

CORBEAU Pierre-Immunologie

COSTES Valérie-Anatomie et cytologie pathologiques

COURTET Philippe-Psychiatrie d'adultes ; addictologie

CYTEVAL Catherine-Radiologie et imagerie médicale

DADURE Christophe-Anesthésiologie-réanimation

DAUVILLIERS Yves-Physiologie

DE TAYRAC Renaud-Gynécologie-obstétrique, gynécologie médicale

DEMARIA Roland-Chirurgie thoracique et cardio-vasculaire

DEMOLY Pascal-Pneumologie ; addictologie

DEREURE Olivier-Dermatologie - vénéréologie

DROUPY Stéphane -Urologie

DUCROS Anne-Neurologie -

FRAPIER Jean-Marc-Chirurgie thoracique et cardiovasculaire

KLOUCHE Kada-Réanimation ; médecine d'urgence

KOENIG Michel-Génétique moléculaire

LABAUGE Pierre- Neurologie

LAFFONT Isabelle-Médecine physique et de réadaptation

LAVABRE-BERTRAND Thierry-Cytologie et histologie

LECLERCQ Florence-Cardiologie

LEHMANN Sylvain-Biochimie et biologie moléculaire

LUMBROSO Serge-Biochimie et Biologie moléculaire

MARIANO-GOULART Denis-Biophysique et médecine nucléaire

MATECKI Stéfan -Physiologie

MEUNIER Laurent-Dermato-vénéréologie

MONDAIN Michel-Oto-rhino-laryngologie

MORIN Denis-Pédiatrie

NAVARRO Francis-Chirurgie générale

PAGEAUX Georges-Philippe-Gastroentérologie ; hépatologie ; addictologie

PETIT Pierre-Pharmacologie fondamentale ; pharmacologie clinique ; addictologie

PERNEY Pascal-Médecine interne ; gériatrie et biologie du vieillissement, médecine générale, addictologie

PUJOL Jean Louis-Pneumologie ; addictologie

PUJOL Pascal-Biologie cellulaire 
PURPER-OUAKIL Diane-Pédopsychiatrie ; addictologie

QUERE Isabelle-Chirurgie vasculaire ; médecine vasculaire (option médecine vasculaire)

SOTTO Albert-Maladies infectieuses ; maladies tropicales

TOUITOU Isabelle-Génétique

TRAN Tu-Anh-Pédiatrie

VERNHET Hélène-Radiologie et imagerie médicale

PU-PH de 2ème classe

ASSENAT Éric-Gastroentérologie ; hépatologie ; addictologie

BERTHET Jean-Philippe-Chirurgie thoracique et cardiovasculaire

BOURDIN Arnaud-Pneumologie ; addictologie

CANAUD Ludovic-Chirurgie vasculaire ; Médecine Vasculaire

CAPDEVIELLE Delphine-Psychiatrie d'Adultes ; addictologie

CAPTIER Guillaume-Anatomie

CAYLA Guillaume-Cardiologie

CHANQUES Gérald-Anesthésiologie-réanimation

COLOMBO Pierre-Emmanuel-Cancérologie ; radiothérapie

COSTALAT Vincent-Radiologie et imagerie médicale

COULET Bertrand-Chirurgie orthopédique et traumatologique

CUVILLON Philippe-Anesthésiologie-réanimation

DAIEN Vincent-Ophtalmologie

DE VOS John-Cytologie et histologie

DORANDEU Anne-Médecine légale -

DUPEYRON Arnaud-Médecine physique et de réadaptation

FESLER Pierre-Médecine interne ; gériatrie et biologie du vieillissement, médecine générale, addictologie

GARREL Renaud -Oto-rhino-laryngologie

GAUJOUX Viala Cécile-Rhumatologie

GENEVIEVE David-Génétique

GODREUIL Sylvain-Bactériologie-virologie ; hygiène hospitalière

GUILLAUME Sébastien-Urgences et Post urgences psychiatriques -

GUILPAIN Philippe-Médecine Interne, gériatrie et biologie du vieillissement ; addictologie

GUIU Boris-Radiologie et imagerie médicale

HAYOT Maurice-Physiologie

HOUEDE Nadine-Cancérologie ; radiothérapie 
JACOT William-Cancérologie ; Radiothérapie

JUNG Boris-Réanimation ; médecine d'urgence

KALFA Nicolas-Chirurgie infantile

KOUYOUMDJIAN Pascal-Chirurgie orthopédique et traumatologique

LACHAUD Laurence-Parasitologie et mycologie

LALLEMANT Benjamin-Oto-rhino-laryngologie

LAVIGNE Jean-Philippe-Bactériologie-virologie ; hygiène hospitalière

LE MOING Vincent-Maladies infectieuses ; maladies tropicales

LETOUZEY Vincent-Gynécologie-obstétrique ; gynécologie médicale

LOPEZ CASTROMAN Jorge-Psychiatrie d'Adultes ; addictologie

LUKAS Cédric-Rhumatologie

MAURY Philippe-Chirurgie orthopédique et traumatologique

MILLET Ingrid-Radiologie et imagerie médicale

MORANNE Olvier-Néphrologie

MOREL Jacques -Rhumatologie

NAGOT Nicolas-Biostatistiques, informatique médicale et technologies de la communication

NOCCA David-Chirurgie digestive

PANARO Fabrizio-Chirurgie générale

PARIS Françoise-Biologie et médecine du développement et de la reproduction ; gynécologie médicale

PASQUIE Jean-Luc-Cardiologie

PEREZ MARTIN Antonia-Physiologie

POUDEROUX Philippe-Gastroentérologie ; hépatologie ; addictologie

PRUDHOMME Michel-Anatomie

RIGAU Valérie-Anatomie et cytologie pathologiques

RIVIER François-Pédiatrie

ROGER Pascal-Anatomie et cytologie pathologiques

ROSSI Jean François-Hématologie ; transfusion

ROUBILLE François-Cardiologie

SEBBANE Mustapha-Anesthésiologie-réanimation

SEGNARBIEUX François-Neurochirurgie

SIRVENT Nicolas-Pédiatrie

SOLASSOL Jérôme-Biologie cellulaire

SULTAN Ariane-Nutrition

THOUVENOT Éric-Neurologie

THURET Rodolphe-Urologie

VENAIL Frédéric-Oto-rhino-laryngologie 
VILLAIN Max-Ophtalmologie

VINCENT Denis -Médecine interne ; gériatrie et biologie du vieillissement, médecine générale, addictologie

VINCENT Thierry-Immunologie

WOJTUSCISZYN Anne-Endocrinologie-diabétologie-nutrition

\section{PROFESSEURS DES UNIVERSITES}

$1^{\text {re }}$ classe :

COLINGE Jacques -Cancérologie, Signalisation cellulaire et systèmes complexes

$\underline{2^{\text {ème }} \text { classe : }}$

LAOUDJ CHENIVESSE Dalila - Biochimie et biologie moléculaire

VISIER Laurent - Sociologie, démographie

\section{PROFESSEURS DES UNIVERSITES - Médecine générale}

$\underline{1^{\text {re }} \text { classe : }}$

LAMBERT Philippe

2ème classe :

AMOUYAL Michel

\section{PROFESSEURS ASSOCIES - Médecine Générale}

DAVID Michel

RAMBAUD Jacques

PROFESSEUR ASSOCIE - Médecine

BESSIS Didier - Dermato-vénéréologie)

PERRIGAULT Pierre-François -Anesthésiologie-réanimation ; médecine d'urgence

ROUBERTIE Agathe - Pédiatrie

Maîtres de Conférences des Universités - Praticiens Hospitaliers

MCU-PH Hors classe

CACHEUX-RATABOUL Valère-Génétique 
CARRIERE Christian-Bactériologie-virologie ; hygiène hospitalière

CHARACHON Sylvie-Bactériologie-virologie ; hygiène hospitalière

FABBRO-PERAY Pascale-Epidémiologie, économie de la santé et prévention

HILLAIRE-BUYS Dominique-Pharmacologie fondamentale ; pharmacologie clinique ; addictologie PELLESTOR Franck-Cytologie et histologie

PUJOL Joseph-Anatomie

RAMOS Jeanne-Anatomie et cytologie pathologiques

RICHARD Bruno-Thérapeutique ; addictologie

RISPAIL Philippe-Parasitologie et mycologie

SEGONDY Michel-Bactériologie-virologie ; hygiène hospitalière

STOEBNER Pierre -Dermato-vénéréologie

\section{MCU-PH de $1^{\text {re }}$ classe}

ALLARDET-SERVENT Annick-Bactériologie-virologie ; hygiène hospitalière

BADIOU Stéphanie-Biochimie et biologie moléculaire

BOUDOUSQ Vincent-Biophysique et médecine nucléaire

BOULLE Nathalie-Biologie cellulaire

BOURGIER Céline-Cancérologie ; Radiothérapie

BRET Caroline -Hématologie biologique

COSSEE Mireille-Génétique Moléculaire

GABELLE DELOUSTAL Audrey-Neurologie

GIANSILY-BLAIZOT Muriel-Hématologie ; transfusion

GIRARDET-BESSIS Anne-Biochimie et biologie moléculaire

LAVIGNE Géraldine-Hématologie ; transfusion

LE QUINTREC Moglie-Néphrologie

MATHIEU Olivier-Pharmacologie fondamentale ; pharmacologie clinique ; addictologie

MENJOT de CHAMPFLEUR Nicolas-Neuroradiologie

MOUZAT Kévin-Biochimie et biologie moléculaire

PANABIERES Catherine-Biologie cellulaire

PHILIBERT Pascal-Biologie et médecine du développement et de la reproduction

RAVEL Christophe - Parasitologie et mycologie

SCHUSTER-BECK Iris-Physiologie

STERKERS Yvon-Parasitologie et mycologie

TUAILLON Edouard-Bactériologie-virologie ; hygiène hospitalière

YACHOUH Jacques-Chirurgie maxillo-faciale et stomatologie 
MCU-PH de $2^{\text {éme }}$ classe

BERTRAND Martin-Anatomie

BRUN Michel-Bactériologie-virologie ; hygiène hospitalière

DU THANH Aurélie-Dermato-vénéréologie

GALANAUD Jean Philippe-Médecine Vasculaire

GOUZI Farès-Physiologie

JEZIORSKI Éric-Pédiatrie

KUSTER Nils-Biochimie et biologie moléculaire

LESAGE François-Xavier-Médecine et Santé au Travail

MAKINSON Alain-Maladies infectieuses, Maladies tropicales

MURA Thibault-Biostatistiques, informatique médicale et technologies de la communication

OLIE Emilie-Psychiatrie d'adultes ; addictologie

THEVENIN-RENE Céline-Immunologie

\section{MAITRES DE CONFERENCES DES UNIVERSITES - Médecine Générale}

COSTA David

FOLCO-LOGNOS Béatrice

\section{MAITRES DE CONFERENCES ASSOCIES - Médecine Générale}

CLARY Bernard

GARCIA Marc

MILLION Elodie

PAVAGEAU Sylvain

REBOUL Marie-Catherine

SEGURET Pierre

\section{MAITRES DE CONFERENCES DES UNIVERSITES}

Maîtres de Conférences hors classe

BADIA Eric - Sciences biologiques fondamentales et cliniques

Maîtres de Conférences de classe normale

BECAMEL Carine - Neurosciences

BERNEX Florence - Physiologie

CHAUMONT-DUBEL Séverine - Sciences du médicament et des autres produits de santé

CHAZAL Nathalie - Biologie cellulaire

DELABY Constance - Biochimie et biologie moléculaire 
GUGLIELMI Laurence - Sciences biologiques fondamentales et cliniques HENRY Laurent - Sciences biologiques fondamentales et cliniques

LADRET Véronique - Mathématiques appliquées et applications des mathématiques

LAINE Sébastien - Sciences du Médicament et autres produits de santé

LE GALLIC Lionel - Sciences du médicament et autres produits de santé

LOZZA Catherine - Sciences physico-chimiques et technologies pharmaceutiques

MAIMOUN Laurent - Sciences physico-chimiques et ingénierie appliquée à la santé

MOREAUX Jérôme - Science biologiques, fondamentales et cliniques

MORITZ-GASSER Sylvie - Neurosciences

MOUTOT Gilles - Philosophie

PASSERIEUX Emilie - Physiologie

RAMIREZ Jean-Marie - Histologie

TAULAN Magali - Biologie Cellulaire

\section{PRATICIENS HOSPITALIERS UNIVERSITAIRES}

CLAIRE DAIEN-Rhumatologie

BASTIDE Sophie-Epidémiologie, économie de la santé et prévention

FAILLIE Jean-Luc-

Pharmacologie fondamentale ; pharmacologie clinique ; addictologie

GATINOIS Vincent-Histologie, embryologie et cytogénétique

HERLIN Christian -Chirurgie plastique ; reconstructrice et esthétique ; brûlologie

HERRERO Astrid-Chirurgie générale

PANTEL Alix-Bactériologie-virologie ; hygiène hospitalière

PERS Yves-Marie-Thérapeutique, médecine d'urgence ; addictologie

PINETON DE CHAMBRUN Guillaume-Gastroentérologie ; hépatologie ; addictologie TORRE Antoine-Gynécologie-obstétrique ; gynécologie médicale 


\section{REMERCIEMENTS}

\section{A MES MAITRES}

Le Professeur Jean Yves LEFRANT, Je vous remercie de me faire l'honneur de présider ce jury de thèse. Des premiers jours de mon internat à ma dernière garde d'interne dont vous étiez le sénior, des conseils scientifiques aux métaphores sportives, vous avez toujours été encourageant et bienveillant.

Le Professeur Pascal COLSON,

Votre présence dans ce jury de thèse est un grand honneur. Merci de m'avoir supporté et trainé dans les couloirs du DAR D dès l'entente de l'acronyme E.C.M.O.

Merci de votre aide et votre soutien dans l'élaboration du projet de simulation qui m'est cher. Merci enfin de vos conseils professionnels. Vous êtes un des rares à nous apprendre la patience et l'humilité.

Le Professeur Guillaume CAYLA,

Votre présence dans ce jury de thèse est un grand honneur. L'insuffisance cardiaque ainsi que les techniques d'assistance cardiaques ont pour moi un grand intérêt. Après avoir embolisé les couloirs du DAR D et de I'USIC montpelliérains, j'espère que nous aurons l'occasion de travailler ensemble à ce sujet avec je l'espère une grande fluidité.

Le Professeur Laurent MULLER,

Merci Laurent, merci pour tout : tes conseils, ton humanité envers les patients, ton aide dans l'élaboration de ce travail...

Tes cours resteront pour moi des moments qui ont marqué mon internat. Travailler à tes cotés va être un immense honneur.

Le Docteur Claire ROGER,

Je te dois énormément, des premiers conseils naïfs de début d'internat aux avis téléphoniques plus précis de remplacements ou de gardes seniorisées. Merci de ton aide dans l'élaboration de la thèse. Merci également pour le magnifique dévouement dont tu fais preuve chaque jour pour l'équipe de réanimation chirurgicale.

Merci également au Docteur Aurélien DAURAT pour ton aide dans l'élaboration de ce travail. II faut s'arrêter à cinq membres de jury sans quoi cela aurait été un honneur de te compter comme membre. 
Merci au Docteur Benjamin LOUART pour sa contribution au travail d'analyse statistique de cette thèse. Merci Professeur JABER pour votre expérience en réanimation vos enseignements.

Merci Professeur CAPDEVILA pour la coordination du DES, pour vos enseignements et pour ces quelques gardes passées ensemble le lundi.

\section{AU MONDE DE LA CARDIOLOGIE POUR AVOIR ACCUEILLI UN ANESTHESISTE DANS LEUR MUR}

Merci à Jean, Etienne et Camille pour m'avoir enseigné les rudiments de l'ETT.

Merci à François ROUBILLE pour m'avoir accueilli 6 mois à I'USIC. Ta rigueur et ta capacité de travail resteront pour moi impressionnantes.

Merci à Kamila et Myriam, les chefs de cliniques qui ont accompagné ce stage.

Merci aux autres PH : Delphine, Fred, Jean Christophe et Stéphane, merci pour vos visites alliant rigueur et plaisanterie.

Et un GRAND MERCI à mes co-internes. Jean, ta gentillesse et ta bonne humeur mise à rude épreuve par

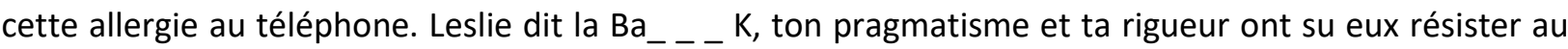
téléphone (ou presque). Merci à tous les deux pour votre coaching en cardio. David puis Pablo, mes compagnons non cardiologues suspectés et surveillés comme moi à la moindre modification du Bisoce, merci pour votre gentillesse et votre bonne humeur.

\section{AUX PERPIGNANAIS}

Pastou, IADE emblématique, Celestin BRU pour ton diplôme d'intubation.

Sébastien KOLLEN et Jean Marie QUINTARD, merci de votre gentillesse et de vos conseils. Vous êtes à l'origine de mon intérêt pour l'ECMO, comme vous le dites, vous m'avez fait « tomber dedans quand j'étais petit ».

A tous les autres PH qui ont marqué mon passage dans la région : Laurent, Arnaud, Vincent, ODM, JAT, Florence, Olivier, Philippe, PGD, Frank, Aline.

\section{AUX NIMOIS}

Merci aux ARC pour tout ce que vous avez fait pour m'aider.

Guillaume, ton pragmatisme, ta facilité à simplifier les choses... et les conseils brico..

Pascal, désormais à PGS, tu fais partie des quelques personnes à avoir profondément marqué mon internat.

Pierrot, merci pour cet intérêt commun à l'ECMO, merci pour ta bienveillance.

Robert, merci pour ton expérience transmise chaque jour.

Patrice, en cinq ans d'internat et de multiples lieux de stages, je n'ai pas trouvé meilleur repas le weekend. Steph, merci pour ton aide dans sepsistrain. 
Merci à toute l'équipe paramédicale, je ne peux citer tout le monde : Delphine, Jérôme, Didier, Marina, Pierrette, Babette, Isabelle, Mag, Ursula, Françoise....

Merci aux équipes du Bloc et tout particulièrement la team Mater modifiée depuis par les différents mercatos : Jean Michel, Mathieu, Cathy, Béa, Laurent.

\section{A L'EQUIPE DE SIMULATION}

Laurent, merci de nous avoir bercé tout petit, et de peu à peu nous laisser voler seul. Olivier et Domi, merci de votre accueil et de votre aide. Encore un grand merci au Pr Colson d'avoir cru en nous et nous avoir soutenu. Merci à Meriem, Norddine, Fabien, le noyau dur, d'avoir fait en sorte que ce projet de simulation se concrétise. Merci à tous les autres qui de près ou de loin, de Nîmes et de Montpellier nous ont aidés et soutenus (Pierre, Geoffroy, Jean Claude, Julien, Fred).

\section{A L'EQUIPE DU DAR D QUI M'A BEAUCOUP EU DANS LES PATTES}

Philippe, merci pour ton expérience que tu nous transmets chaque jour.

Jacob, merci pour ta bonne humeur, pour ton énorme expérience en assistance, et .... pour ta participation sans rechigner aux soirées.

Nordine, je te dois énormément, merci pour ton aide, ton écoute, tes conseils à persévérer pour faire ce qu'il plait. Et sans oublier ces discussions de gardes qui ont quand même était la base du projet de simulation.

Marc merci pour ta bonne humeur, pour ton expérience que tu partages avec plaisir.

Meriem, je te dois énormément et sur beaucoup de points, merci pour tout : tes enseignements, la simulation, les avis mais également ta gentillesse et ta disponibilité.

Gégé, merci pour tes enseignements en échographie, et comme Jacob ta participation indéfectible aux soirées.

Merci également à tous les autres anesthésistes avec qui j'ai moins partagé mais qui m’ont également beaucoup apporté : Michelle, Philippe, Denis, Marion, Pierre, Marine.

ET UN GRAND merci aux perfus : Jean Claude, merci pour ta légendaire bonne humeur ta soif de transmettre et cette passion pour ton métier. Fabien, merci pour ces heures passées à bricoler ce simulateur, ta gentillesse, ta disponibilité. Fred, merci pour cette bonne humeur que tu nous transmets, tes conseils et ta disponibilité. Julien, la force tranquille, le dernier arrivé, merci de ton aide pour la simulation. 


\section{A LA REANIMATION MEDICALE}

Merci à toute l'équipe de la réanimation médicale pour leur accueil, leur écoute ... et parfois ces longs débats, et ces observ infinissables ... Professeur JONQUET, Professeur KLOUCHE et Professeur JUNG, Vincent, Philippe, Laura, Noémie, Delphine, Liliane, Corine, Aurel, Sonia, Valérie.

Merci à mes co-internes qui ont égayé ce stage. Merci à Yassir, Mélanie, Mathieu, Max, Benoit, lleana, Fanny, Féie, Cédric.

Merci également à tant d'autres dans les différents DAR qui m'ont beaucoup apporté : Olivier en ALR Jonathan en traumato, Anne Cha, Christophe, Pablo, Isa, Olivier, Laurent, Christelle, Maud, Christine en pédiatrie.

\section{A L'EQUIPE DE LA CLINIQUE DES FRANCISCAINES,}

Merci pour leur excellent accueil.

Guillaume, merci pour ces longues discussions, tes enseignements, ta disponibilité, ton enthousiasme.

David, merci pour ta gentillesse, ton efficacité dans le travail, et cette foi en l'EBM.

Damien, merci pour cette rigueur, ces conseils et ta disponibilité.

Rick, merci d'avoir accepté de faire cobaye en simulation d'ECMO.

Antoine, les Olivier, Jean François, Isabelle, Monsieur Julien pour votre expérience, votre sens clinique que vous m'avez transmis.

Toufic, merci pour ta gentillesse, ton humanisme en réanimation.

Et à tous les autres que je ne cite pas.

\section{A MA FAMILLE}

A mes parents,

La loyauté, la servitude, la dévotion pour son prochain sont les mots qui vous caractérisent. Je vous admire tous les jours. Si je vis cette aventure de médecine, c'est intégralement grâces à vous. La ténacité à toujours m'expliquer que si les autres y arrivent, pourquoi pas moi. Ces valeurs humanistes que vous m'avez enseignées, je ne les oublierai jamais.

A Elsa, ma chérie,

Parfois je me demande comment tu supportes les inconvénients inhérents à notre métier. D'abord l'ECN puis l'internat avec ses gardes et maintenant ce rôle de senior avec les responsabilités et les doutes qui lui incombent, ces repas au silence pesant lorsque l'on ressasse une prise en charge à tort ou à raison discutable... Merci pour ton soutien, tu me rends plus fort, tu nous rends plus fort. Merci pour ton aide dans la correction de ce travail. 
A mes ainés qui ne sont plus là,

Ma grand-mère, je t'ai peu connue mais là encore les valeurs de travail, fierté, robustesse et générosité été présentes.

Franc et Henriette : je ne saurais jamais si c'est la sagesse de l'âge mais vous avez été d'un soutien indéfectible ne marquant jamais en ma présence l'ombre d'un doute à la réussite d'un quelconque examen et par voie de fait ne marquant aucune surprise à l'annonce de sa réussite mais un simple « je le savais ".

A ma tante et mon oncle,

Je vous dois également beaucoup, soutien de toujours. Des noëls passés en famille, à l'aide dans les travaux d'aménagement, vous avez toujours été présents. Votre histoire, faite de joies et de tristesses, les valeurs de famille, de travail et de bienveillance que vous portez sont pour moi des piliers importants.

A mes cousins,

Laurent, que de souvenirs dans l'insouciance de l'adolescence, ces parties de braconnage, ces journées d'hiver s'entrainant au pilotage sur neige. A Sophie qui partage sa vie et le rend heureux.

Sophie, ma cousine, avec qui je garde le souvenir d'une enfance heureuse, complice et insouciante. La vie ne nous a malheureusement pas permis de nous connaitre adulte mais tu gardes une place importante dans mon cœur.

Marine, ma cousine montpelliéraine. Merci pour ces discussions que l'on peut avoir. Tu as connu mes doutes et mes joies. Je sais que je peux compter sur toi.

A mes petits cousins TITI et Margaux.

A Simone GEMINARD, je te dois énormément. Je n'oublierai jamais ce que tu as fait pour moi. Le concours de première année, $c^{\prime}$ est en grande partie à toi que je le dois. Tu as fait preuve de générosité, tu m'as appris la persévérance, comment canaliser mes doutes, comment aller à l'essentiel. Je crois bien qu'il s'agit de la clef indispensable de la réussite.

Merci également à tous les autres membres de ma famille qui comptent beaucoup. Je ne peux citer tout le monde.

\section{A MES AMIS D’ENFANCE}

Pierre, Laure, Elena, Christophe, Seb et John. De l'école primaire à ce jour, vous avez été constamment présents. Vous avez toujours été là pour moi malgré les kilomètres et les inconvénients du métier. Je ne I'oublierai jamais. Les valeurs de travail, de servitude, d'amitié qui nous ont été enseignées dans la petite 
enfance et que vous me rappelez à chacun de mes retours aux pays fond partie de mes piliers. Les (rares) sorties à moto, les soirées sont également des moments de décompression inestimables.

\section{A MES AMIS D’EXTERNATS}

Corentin, merci pour ta droiture légendaire, tes valeurs, tes racines campagnardes. Le tableau serait presque parfait si tu n'étais pas ardéchois.

Peete, merci pour ces délires, ta gentillesse, ta disponibilité et notre complicité. Même si tes racines catalanes t'on rappelées à l'ordre et éloignées de la maison mère, j'espère que l'on se verra régulièrement.

Thomas Roland (TR), merci pour ces soirées inoubliables.

Mathieu, Simon, Najib, Arnal, J, Guiz et Greg, merci pour tous ces moments passés ensemble.

Mathilde, Julie et Léa, merci pour votre soutien lors de la D4. Merci de votre générosité.

\section{A MES AMIS D'INTERNAT}

Yassir, merci pour ta disponibilité, ces discussions nocturnes interminables...

Merci à tous mes autres copains de promo : Geoffrey, Tim , Lucie , Yoyo, Guigui, Céline, Julien, Simon , Severin, Clément , Guillaume, Benjamin , Habib et Maxime. Merci pour ces semestres passés ensemble, pour ces séminaires et pour votre soutien.

Kevin, Clément, Jerem, Jean Clément et Medhi, pas la même génération mais vous êtes brillamment sortis du $10 \mathrm{ml} / \mathrm{kg}$ de vT. Merci les potos, ce stage au DAR D et vos conseils de premiers remplacements, je ne les oublierai pas.

Et merci à tous les autres qui ont compté pour moi et que je ne peux citer, mes coexternes de Montpellier, tous mes co-internes dans les différents services, tous mes chefs de cliniques, les infirmiers, secrétaires et aides-soignants ... 


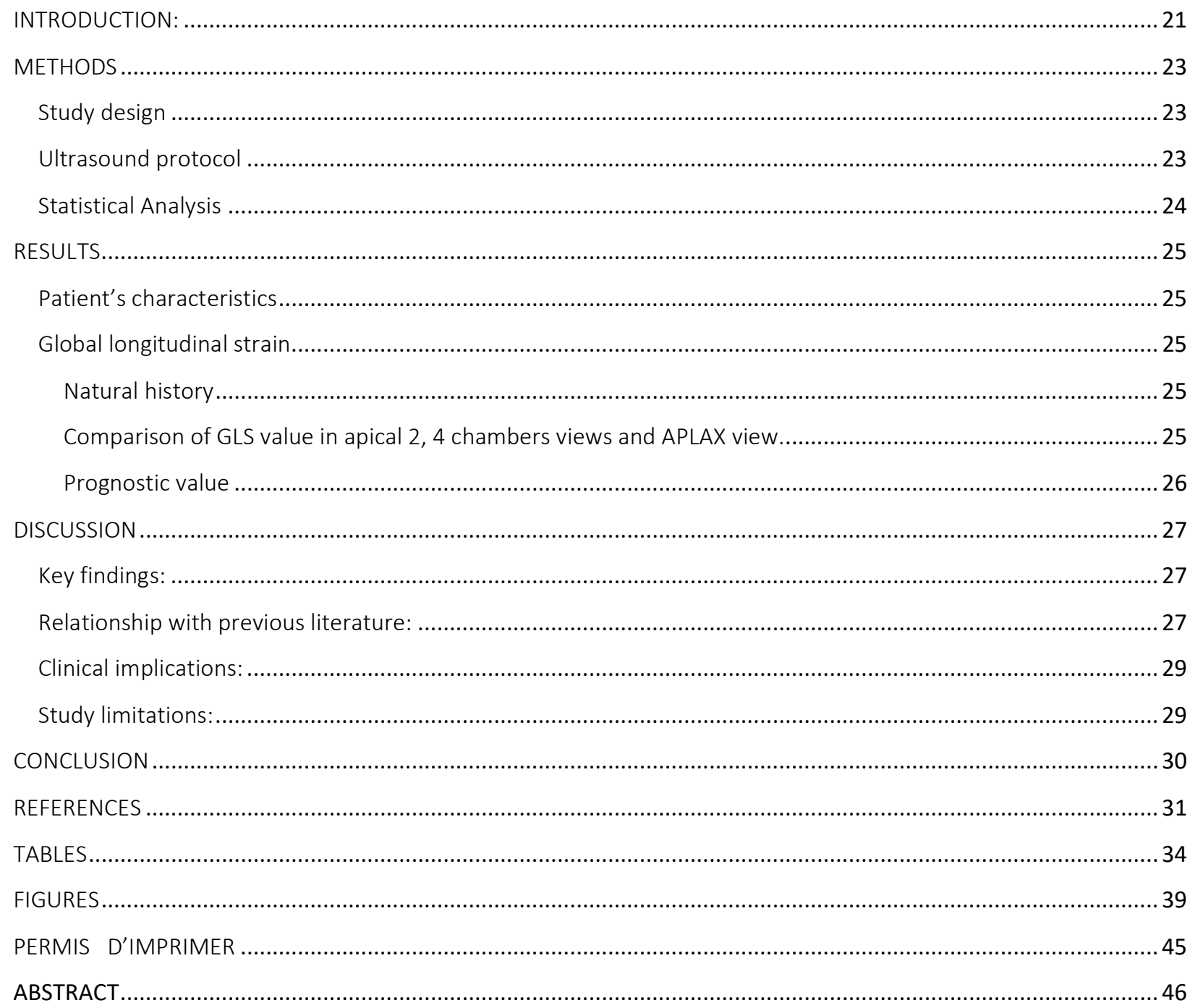




\section{Prognostic value and time course evolution}

\section{of left ventricular Global Longitudinal Strain in septic shock:}

\section{An exploratory prospective study}

\section{INTRODUCTION:}

Septic cardiomyopathy (SCM) is observed in up to $40 \%$ of patients with septic shock (1). This clinical entity has been firstly described by Parker in the 80 's, who suggested that left ventricle ejection fraction (LVEF) was paradoxically higher in non-survivors of septic shock (2). Subsequent studies did not confirm these results and a meta-analysis did not show any link between LVEF and mortality in septic population(3).

The main mechanism for high LVEF is profound inflammatory-induced vasoplegia, which decreases cardiac afterload and artificially increases LVEF. Severe vasoplegia and high dose of vasopressors requirement is associated to poor outcome, leading to refractory shock (4). Thus, LVEF seems too dependent on loading conditions to be a reliable marker of intrinsic myocardial contractility in septic shock (5)(6). It could be hypothesized that other echocardiographic markers of intrinsic contractility could be interesting to detect SCM. The use of conventional echocardiography is now widely recommended at bedside for non-invasive hemodynamic assessment in intensive care unit (ICU) patients, but the clinical usefulness of myocardial strain assessment remains unclear(7) (8).

Real-time speckle-tracking imaging is now widely available on echocardiography machine at bedside. This technology allows to assessing myocardial deformation over the cardiac cycle. Myocardial deformation is quantified by measuring global longitudinal strain (GLS) as an index of systolic function. In cardiology, this techniques have been validated after comparison with gold standard techniques such as magnetic resonance imaging (9). Myocardial longitudinal strain assessment is interesting due to a fast learning curve, an automatized measurement and a good inter and intra observer reproducibility (10) (11) (12). Initial experimental and humans data suggest that initial GLS could be a better and earlier marker of SMC than LVEF (13)(6). The impact of GLS alterations on prognosis remains debated. An altered GLS value at admission (> - $13 \%$ ) has been shown to independently predict mortality in septic shock (14) (15) whereas one study 
did not confirm the association between GLS and prognosis (16). Two issues remain unclear about strain in ICU. First, the evolution of GLS over time can be of clinical importance but has been poorly described (16). Second, the possibility to simplify strain assessment in ICU patients seems necessary as previous reports have suggested a feasibility ranging from $42 \%$ to $77 \%$ (6)(17). This variable feasibility is related to difficult assessment of GLS because of limited echogenicity for anterior wall in 2 and 3 chambers views (18). Theoretically, GLS should be assessed in 2, 3 and 4 chambers views and subsequently averaged. This is difficult and time consuming in ICU patients, even if automatic real-time measurement simplifies the procedure.

To bring answers to the two last issues, the present study was aimed at:

1) Describing the natural history of left ventricle (LV) GLS during septic shock.

2) Evaluating the correlation between average LV GLS measured in apical two, three or four chambers and a single value of LV GLS recorded in a single 4 chamber view (Single Longitudinal Strain, SLS), which is easier and more rapid to assess. We hypothesized that SCM is a global heart disease and that there would be no difference between average LV GLS and SLS.

3) Studying the correlation between strain value and prognosis according to initial LVEF. 


\section{METHODS}

\section{Study design}

A prospective, observational cohort study was conducted in a single medico-surgical ICU of the Nimes University hospital from February 2016 to march 2018(19).

According to the French law, the study protocol was approved by the local Ethics Committee (IRB/16.02.07) that waived written inform consent. Written information was given to the patient or his/her relatives who could refuse to participate.

All patients aged $\geq 18$ years admitted for a diagnostic of septic shock were enrolled in the present study. Septic shock was defined by clinical evidence of sepsis with persisting hypotension requiring vasopressors to maintain $\mathrm{MAP} \geq 65 \mathrm{~mm} \mathrm{Hg}$ and a serum lactate level $>2 \mathrm{mmol} / \mathrm{L}$ $(18 \mathrm{mg} / \mathrm{dL})$ despite adequate volume resuscitation, according to current guidelines (20).

Non-inclusion criteria were age $<18$ years, pregnancy, severe valvular disease, ischemic heart disease with known past history of ischemic sequelae, others cardiomyopathies, atrial fibrillation, patients in whom LV walls could not be evaluated through standard apical views.

The following variables were recorded: age, gender, body mass index, past medical history, septic origin, vasopressor doses, mechanical ventilation use, ICU length of stay and 30 days mortality (21). The severity of organ failure was assessed with the simplified acute physiology score (SAPS) Il at admission (22).

All patients were treated according to international guidelines septic shock management (23).

\section{Ultrasound protocol}

The echocardiographic examination was firstly performed during the first 24 hours after ICU admission (T1) and repeated once between day 3 and day 5 (T2) then once between day 6 and day 8 (T3).

All echocardiographic exams were performed by an expert in echocardiography using a Vivid S70 ultrasound device (GE Healthcare). Two-dimensional apical two-chamber, four-chamber and long-axis views (2C, 4C and ALAX) were recorded. Speckle tracking analysis was performed during a post treatment analysis after recording the echocardiographic loops. Briefly, analysis of LV myocardial deformation is performed from 2-dimensional gray-scale loops by automatic tracking of myocardial speckles after manual selection of landmark points using apical views of the left ventricle. GLS was calculated as the average of negative peak of longitudinal strain from each of 
the 18 LV segments from the $2 \mathrm{C}, 4 \mathrm{C}$ and ALAX views. Formula: $\mathrm{GLS}=(\mathrm{LS}-\mathrm{Ld}) / \mathrm{Ld})($ annex 1$)$ (where Ls= systolic length Ls= diastolic Length)

The main objective of the present study was to report the course of GLS throughout sepsis. Therefore we compare GLS with LVEF in the overall population and in two distinct population: LVEF $\geq 45 \%$ and LVEF $<45 \%$.

Single Longitudinal Strain (SLS) was defined as longitudinal strain measured on 4 chambers view Left ventricle volumes and LVEF were calculated using the Simpson's method (24). The E and A waves velocities and $E$ wave deceleration time were measured using pulsed wave (PW) Doppler in the mitral inflow at the tip of the valve visualized by an apical 4-chambers view. Early diastolic tissue wave velocity of the lateral mitral annulus ( $E^{\prime}$ wave velocity) was measured in the apical $4 C$ view using PW tissue Doppler. Then we calculated the E/E' ratio. Velocity Time Integral (VTI) of the left ventricular outflow tract we measured on an apical five-chambers view. For the right ventricular (RV) evaluation the dilatation is assessed in four chambers with focus on the right ventricle (25). The longitudinal contractility was assessed by the tricuspid annular plane systolic excursion (TAPSE) by using M mode on a 4-chambers view.

At the time of echocardiographic examination, were also recorded clinical parameters: heart rate, systolic, diastolic, and mean blood pressure, as well as biologic parameters: $\mathrm{pH}, \mathrm{pO}_{2}, \mathrm{pCO}_{2}$, base excess, bilirubin, lactates, troponins, platelets and creatinine.

\section{Statistical Analysis}

Descriptive statistics were reported using median and interquartile range (25th to 75th percentile) for continuous variables. For categorical variables, frequencies and proportions were given. To assess the change between $\mathrm{T} 1$ and $\mathrm{T} 2$ for the average GLS, the LVEF, the PAS, the heart rate, lactates, mitral E wave, E/Ea ratio and the sub aortic VTI, we used a paired Student's t-test. To study the relation between several covariates at baseline (average GLS, LVEF, MAP, heart rate, lactates, SAPSII, sex, age and troponin) and survival we used a Cox proportional hazards model. The hazard ratio was computed for each variable in an univariate way. Finally, to assess the agreement between average GLS and four-chamber GLS we used the Bland and Altman method by computing the mean difference and its $95 \%$ confidence interval and limits of agreement defined as mean difference \pm 1.96 SD. A $p$ value of 0.05 was considered as significant. All statistical analysis were performed using R software (version 3.3.2). 


\section{RESULTS}

\section{Patient's characteristics}

Among 40 initially consecutive patients, 3 patients were excluded: two patients had previous ischemic cardiomyopathy with anterior sequelae and one patient had an atrial fibrillation. Table 1 summarizes the main clinical characteristics of 37 included patients. All patients reached the targeted mean arterial blood pressure $>65 \mathrm{mmHg}$ as usually recommended (8). No patient was lost for follow up. No patient died during study protocol. Nine patients (24.3\%) died during the month after ICU admission. The site of infection is shown in Table 1.

\section{Hemodynamic and biological parameters: (Tables 2 and 3 )}

Between T1 and T2 exams, a decrease in heart rate over time was observed, and increases in systolic blood pressure and pulse pressure. Considering the echocardiography parameters, ITV increased between $\mathrm{T} 1$ and $\mathrm{T} 2$ whereas others parameters remained stable (Table 2).

Table 3 shows the biological parameters with a decrease in arterial lactate and an increase in base excess between $\mathrm{T} 1$ and $\mathrm{T} 2$

\section{Global longitudinal strain}

Natural history

GLS could be calculated in 36,23 and 9 patients at T1, T2 and T3, respectively. Baseline GLS was impaired (>-13\%) in 23 patients (65.7\%). In overall population GLS, improved throughout the study whereas FEVG did not change (figure1).

In the subgroup of patients with LVEF $<45 \%$ (7 patients), LVEF and GLS evolution over time was similar (Figure 2a and 2b). The initial GLS was impaired in low LVEF Group compared to LVEF $\geq 45 \%$ group $(-9.6$ vs $-13.3 \%, p=0.01)$. In this sub group, both GLS and LVEF significantly improved at T2 (GLS: -9.6 vs $-14.3 \% \mathrm{p}=0.008$ ) (LVEF: $29 \%$ vs $50 \% \mathrm{p}=0.01$ ) (Figure 2a 2b).

\section{Comparison of GLS value in apical 2, 4 chambers views and APLAX view.}

The comparison between mean GLS values in 2, 3 and 4 chambers view and the averaged GLS are reported in Figure 3. The Bland and Altman method shows a good agreement between GLS and SLS: For T1 exams ( $n=35)$ mean of differences -0.04 [-0.7; 0.62]; For T2 exams $(n=23)$ mean of differences $0.1[-0.96 ; 1.16]$ Figure4 a et $4 b$. 
Prognostic value

The course of GLS in survivors and non-survivors is shown in table 4. Only Initial GLS alteration was significantly associated with 30-day mortality rate (-9.1 in non-survivors at day 30 vs $-11 \%$ in survivors OR $1,44[1.13 ; 1.85], p$ 0,004). 


\section{DISCUSSION}

\section{Key findings}

Global longitudinal strain was altered at day 1 of septic shock and significantly improved over time, until normalization. On the opposite, conventional methods for LV systolic function assessment as LVEF and S wave velocity were not affected by the natural course of sepsis. As a second result, GLS values were comparable whatever the echocardiographic view $(2,4$ chambers and APLAX views) considered, suggesting that a single measurement in 4 chambers view (SLS) is sufficient at bedside. Finally, our study confirms that GLS alterations at day 1 are associated with poor outcome.

\section{Relationship with previous literature}

Septic cardiomyopathy has been well reported during septic shock(26). Recent data suggest that diastolic dysfunction is more frequent and more associated with prognosis than systolic dysfunction (27) (28). Moreover, supranormal systolic function (hyperkinetic state, defined as an LVEF > $60 \%$ ) is associated with worse prognosis (6). Systolic function is usually assessed by echocardiography, by measuring conventional LVEF. Diastolic function is assessed by E' wave velocity recording (16). Global longitudinal strain has two theoretical advantages over conventional TTE myocardial function assessment. First, GLS is correlated to both systolic and diastolic function during sepsis (16). Second, GLS can be altered while LVEF remains preserved. Therefore, GLS could be a better and earlier indicator of systolic dysfunction than LVEF. In an experimental model of $E$ coli-induced sepsis in anesthetized and ventilated piglets, Hestenes et al (13) reported a significant linear alteration of LV GLS after $E$ coli infusion (from $-17.2 \% \pm 2.8 \%$ to $-12.3 \% \pm 3.2 \%, p=0.04)$ on a study period of 240 minutes. Right ventricle GLS was also significantly altered (from $-24.2 \% \pm 4.1 \%$ to $-16.9 \% \pm 5.7 \%, p=0.02$ ). In this model, LVEF remained unaltered (from $48 \% \pm 7 \%$ to $49 \% \pm 5 \%$ over the study period, $p=0.4$ ). The conclusion was that GLS helps at assessing myocardial dysfunction during sepsis while LVEF assessment may underestimate this diagnosis. Analogous results have been obtained in humans. The evolution of strain has been studied in the first day of sepsis and septic shock (29). At baseline, GLS was abnormal both in sepsis and septic shock patients (-15\%). At 24 hours, GLS was significantly impaired only in septic shock patients $(-13 \%$ at $\mathrm{H} 24)$ but unchanged in sepsis group $(-17 \%$ at $\mathrm{H}$ 24), suggesting a more profound myocardial impairment during septic shock than during sepsis and a link between severity and GLS. In this study, in contrast to GLS, LVEF remained normal in both groups. This study showed that rapid and profound change in GLS value can occur during the first 24 hours of septic shock, without concomitant alterations of LVEF. In our study, patients 
were mainly analyzed at the end of the first 24 hours of septic shock, explaining why a very poor value of GLS (-11.7 \%) was reported at day 1, close to value reported by Shahul et al (29). In a case control study involving 62 septic shock, $\mathrm{Ng}$ et al showed that septic patients had a greater degree of myocardial dysfunction measured by GLS as compared to non-septic patients (-14.5\% vs $-18.3 \%, p<0.001$, respectively)(30). In this study, LVEF was normal and not different between the two groups ( $59 \%$ in the sepsis group vs $61 \%$ in the control group, $P=0.169$ ). In a large study involving 132 septic patients, GLS was altered in 70\% of patients, whatever the value of LVEF (6). Similarly, our results confirm that GLS is altered during sepsis whereas LVEF remains normal as well as $\mathrm{S}$ wave velocity, a classical marker of systolic function, which is not altered over time in the present study (Table 2). Additionally, the present results show that, in the whole population, GLS improves over time while LVEF and S wave velocity remain stable in the natural course of sepsis. In the sub group of patients with altered LVEF at baseline, both LVEF and strain improved from T1 to T2. In the sub group of patients with altered LVEF, the clinical interest of strain to characterize SCM is less clear than for patients with normal LVEF

Because both preload and afterload are decreased at the early phase of sepsis, LVEF can be artificially high and usually normal in both survivors and non-survivors (27). Theoretically, GLS should be less affected by loading conditions than LVEF (31) because it does not depend on ventricular volume (32) (16). However, recent studies challenge the concept that myocardial strain is independent from loading conditions. Nafati et al. recently reported that, in preload dependent patients, strain can move from pathological to normal value after fluid challenge $(-13.3 \pm 3.5$ to $-18.4 \% \pm 4.5(p<0.01)(33)$. This means that the improvement of GLS over time during sepsis observed in the present report could be partially due to improvement of preload conditions during fluid resuscitation. We did not observe any changes in classical static echocardiography preload indicators (E wave velocity, $E / E^{\prime}$ ratio, E/A ratio) (6) over time while we report a significant improvement of sub aortic VTI (reflecting stroke volume) over the study period. Therefore, we hypothesize that GLS improvement over time is rather due to favorable myocardial function evolution during sepsis rather than to preload increase. Our results slightly differ from those obtained by De Geer et al. in terms of improvement timing (16). This group reported a significant impairment of GLS from day 1 to day 3-4 and a return to baseline value at the end of sepsis. Both studies suggest that GLS is affected by sepsis and subsequently improves.

While a new assessment tool becomes available at bedside, it seems crucial to evaluate both its reliability and its feasibility in order to implement this tool in clinical practice. Thus, the ability for 
an ICU physician to rapidly and easily perform GLS in ICU patients at bedside is of importance. Boissier et al. (6) recently reported a poor feasibility (<50\%) of GLS during septic shock. Difficult assessment of GLS is classically due to limited echogenicity for anterior wall in 2 and 3 chambers views(18). Moreover, GLS assessment in 2 and 4 chambers views and APLAX incidence is time consuming, even with machines allowing automatized real time analysis. Interestingly, our results suggest that GLS values are not statistically different in these three incidences. These findings support the hypothesis that septic cardiomyopathy is a global disease, which equally affects all LV myocardial segments. Therefore, GLS analysis from a single incidence (4 chambers view) appears to be good enough at bedside to detect SCM.

Our study shows that only GLS value at the early phase of sepsis is associated with mortality whereas subsequent values are not related to prognosis. The prognosis value of early GLS confirms the conclusions of previous reports (14) (15). In a recent metanalysis, Sanfilippo et al. demonstrated worse GLS values associated with higher mortality in patients with severe sepsis or septic shock (34) These results highlight that clinical usefulness of GLS to predict outcome deserve further investigation.

\section{Clinical implications:}

The present report shows that GLS is profoundly depressed at the early phase of septic shock and subsequently improves. This early value of GLS better reflect SCM than LVEF. The second implication is that a single GLS measurement on 4 chambers view indirectly reflects the whole myocardial strain. This can be a simplest method to evaluate GLS at bedside. Finally, our results support previous results showing that initial (Day 1) GLS is associated with outcomes. This can help to detect a more severe population, even when LVEF is normal.

\section{Study limitations:}

This study has several limitations. First, this is a single centre study. Second, a small number of subjects were included, limiting the statistical power of the study. The third limitation is the high number of missing data at day 8. 


\section{CONCLUSION}

Myocardial strain is depressed at the early phase of septic shock and improves over time. Despite a profound alteration of strain, LVEF remains normal and does not reflect severity of SCM and its subsequent evolution. A single measurement of GLS in a 4 chambers view appears sufficient at bedside as compared to complete myocardial evaluation (2, 4 chambers and APLX views). Finally, our results support the idea that GLS at day 1 (and not subsequent days) is associated with patient outcome. 


\section{REFERENCES}

1. Ehrman RR, Sullivan AN, Favot MJ, Sherwin RL, Reynolds CA, Abidov A, et al. Pathophysiology, echocardiographic evaluation, biomarker findings, and prognostic implications of septic cardiomyopathy: a review of the literature. Crit Care Lond Engl. 2018 May 4;22(1):112.

2. Parker MM, Shelhamer JH, Bacharach SL, Green MV, Natanson C, Frederick TM, et al. Profound but reversible myocardial depression in patients with septic shock. Ann Intern Med. 1984 Apr;100(4):483-90.

3. Huang SJ, Nalos M, McLean AS. Is early ventricular dysfunction or dilatation associated with lower mortality rate in adult severe sepsis and septic shock? A meta-analysis. Crit Care Lond Engl. 2013 May 27;17(3):R96.

4. Martin C, Medam S, Antonini F, Alingrin J, Haddam M, Hammad E, et al. NOREPINEPHRINE: NOT TOO MUCH, TOO LONG. Shock Augusta Ga. 2015 Oct;44(4):305-9.

5. Vieillard-Baron A. Septic cardiomyopathy. Ann Intensive Care. 2011 Apr 13;1:6.

6. Boissier F, Razazi K, Seemann A, Bedet A, Thille AW, de Prost N, et al. Left ventricular systolic dysfunction during septic shock: the role of loading conditions. Intensive Care Med. 2017 May;43(5):633-42.

7. Seymour CW, Rosengart MR. Septic Shock: Advances in Diagnosis and Treatment. JAMA. 2015 Aug 18;314(7):708-17.

8. Cecconi M, De Backer D, Antonelli M, Beale R, Bakker J, Hofer C, et al. Consensus on circulatory shock and hemodynamic monitoring. Task force of the European Society of Intensive Care Medicine. Intensive Care Med. 2014 Dec;40(12):1795-815.

9. Amundsen BH, Helle-Valle $\mathrm{T}$, Edvardsen $\mathrm{T}$, Torp $\mathrm{H}$, Crosby J, Lyseggen $\mathrm{E}$, et al. Noninvasive myocardial strain measurement by speckle tracking echocardiography: validation against sonomicrometry and tagged magnetic resonance imaging. J Am Coll Cardiol. 2006 Feb 21;47(4):789-93.

10. Negishi T, Negishi K, Thavendiranathan P, Cho G-Y, Popescu BA, Vinereanu D, et al. Effect of Experience and Training on the Concordance and Precision of Strain Measurements. JACC Cardiovasc Imaging. 2017 May;10(5):518-22.

11. Dandel M, Lehmkuhl H, Knosalla C, Suramelashvili N, Hetzer R. Strain and strain rate imaging by echocardiography - basic concepts and clinical applicability. Curr Cardiol Rev. 2009 May;5(2):133-48.

12. Mentz RJ, Khouri MG. Longitudinal Strain in Heart Failure With Preserved Ejection Fraction: Is There a Role for Prognostication? Circulation. 2015 Aug 4;132(5):368-70.

13. Hestenes SM, Halvorsen PS, Skulstad H, Remme EW, Espinoza A, Hyler S, et al. Advantages of strain echocardiography in assessment of myocardial function in severe sepsis: an experimental study. Crit Care Med. 2014 Jun;42(6):e432-440.

14. Chang W-T, Lee W-H, Lee W-T, Chen P-S, Su Y-R, Liu P-Y, et al. Left ventricular global longitudinal strain is independently associated with mortality in septic shock patients. Intensive Care Med. 2015 Oct;41(10):1791-9.

15. Innocenti F, Palmieri V, Guzzo A, Stefanone VT, Donnini C, Pini R. SOFA score and left ventricular systolic function as predictors of short-term outcome in patients with sepsis. Intern Emerg Med. 2018 Jan;13(1):518.

16. De Geer L, Engvall J, Oscarsson A. Strain echocardiography in septic shock - a comparison with systolic and diastolic function parameters, cardiac biomarkers and outcome. Crit Care Lond Engl. 2015 Mar 26;19:122. 
17. Nafati C, Lançon V, Blasco V, Zieleskiewicz L, Harti K, Wiramus S, et al. Two-dimensional-strain echocardiography in intensive care unit patients: A prospective, observational study. J Clin Ultrasound JCU. 2016 Jul 8;44(6):368-74.

18. Marwick TH, Leano RL, Brown J, Sun J-P, Hoffmann R, Lysyansky P, et al. Myocardial strain measurement with 2-dimensional speckle-tracking echocardiography: definition of normal range. JACC Cardiovasc Imaging. 2009 Jan;2(1):80-4.

19. Authors: Marc Leone, Jean-Michel Constantin, Claire Dahyot, Caroline Duracher, Arnaud Friggeri, Olivier Joannes Boyau, Olivier Langeron, Matthieu Legrand, Yazine Mahjoub, Sébastien Mirek, Ségolène Mrozek, Laurent Muller, Jean-Christophe Orban, Christophe Quesnel, Antoine Virat, Xavier Capdevila. French intensive care unit organization [Internet]. [cited 2018 Sep 18]. Available from: Anesthesiology and Intensive Care Medicine

20. Singer M, Deutschman CS, Seymour CW, Shankar-Hari M, Annane D, Bauer M, et al. The Third International Consensus Definitions for Sepsis and Septic Shock (Sepsis-3). JAMA. 2016 Feb 23;315(8):801-10.

21. Nouira H, Ben Abdelaziz A, Kacem M, Ben Sik Ali H, Fekih Hassen M, Ben Abdelaziz A. Which indicators used to assess quality performance in Intensive Care Units? A systematic review of medical literature. Anaesth Crit Care Pain Med. 2018 Jul 25;

22. Le Gall JR, Lemeshow S, Saulnier F. A new Simplified Acute Physiology Score (SAPS II) based on a European/North American multicenter study. JAMA. 1993 Dec 22;270(24):2957-63.

23. Rhodes A, Evans LE, Alhazzani W, Levy MM, Antonelli M, Ferrer R, et al. Surviving Sepsis Campaign: International Guidelines for Management of Sepsis and Septic Shock: 2016. Intensive Care Med. 2017 Mar;43(3):304-77.

24. Lang RM, Bierig M, Devereux RB, Flachskampf FA, Foster E, Pellikka PA, et al. Recommendations for chamber quantification: a report from the American Society of Echocardiography's Guidelines and Standards Committee and the Chamber Quantification Writing Group, developed in conjunction with the European Association of Echocardiography, a branch of the European Society of Cardiology. J Am Soc Echocardiogr Off Publ Am Soc Echocardiogr. 2005 Dec;18(12):1440-63.

25. Harjola V-P, Mebazaa A, Čelutkienè J, Bettex D, Bueno H, Chioncel O, et al. Contemporary management of acute right ventricular failure: a statement from the Heart Failure Association and the Working Group on Pulmonary Circulation and Right Ventricular Function of the European Society of Cardiology. Eur J Heart Fail. 2016 Mar;18(3):226-41.

26. Beesley SJ, Weber G, Sarge T, Nikravan S, Grissom CK, Lanspa MJ, et al. Septic Cardiomyopathy. Crit Care Med. 2018 Apr;46(4):625-34.

27. Sanfilippo F, Corredor C, Fletcher N, Landesberg G, Benedetto U, Foex P, et al. Diastolic dysfunction and mortality in septic patients: a systematic review and meta-analysis. Intensive Care Med. 2015 Jun;41(6):1004-13.

28. Sanfilippo F, Corredor C, Arcadipane A, Landesberg G, Vieillard-Baron A, Cecconi M, et al. Tissue Doppler assessment of diastolic function and relationship with mortality in critically ill septic patients: a systematic review and meta-analysis. Br J Anaesth. 2017 Oct 1;119(4):583-94.

29. Shahul S, Gulati G, Hacker MR, Mahmood F, Canelli R, Nizamuddin J, et al. Detection of Myocardial Dysfunction in Septic Shock: A Speckle-Tracking Echocardiography Study. Anesth Analg. 2015 Dec;121(6):1547-54.

30. Ng PY, Sin WC, Ng AK-Y, Chan WM. Speckle tracking echocardiography in patients with septic shock: a case control study (SPECKSS). Crit Care Lond Engl. 2016 May 14;20(1):145. 
31. Pulido JN, Afessa B, Masaki M, Yuasa T, Gillespie S, Herasevich V, et al. Clinical Spectrum, Frequency, and Significance of Myocardial Dysfunction in Severe Sepsis and Septic Shock. Mayo Clin Proc. 2012 Jul;87(7):620-8.

32. Russo C, Jin Z, Homma S, Rundek T, Elkind MSV, Sacco RL, et al. Relationship of multidirectional myocardial strain with radial thickening and ejection fraction and impact of left ventricular hypertrophy: a study in a community-based cohort. Echocardiogr Mt Kisco N. 2013 Aug;30(7):794-802.

33. Nafati C, Gardette M, Leone M, Reydellet L, Blasco V, Lannelongue A, et al. Use of speckle-tracking strain in preload-dependent patients, need for cautious interpretation! Ann Intensive Care. 2018 Feb 21;8(1):29.

34. Sanfilippo F, Corredor C, Fletcher N, Tritapepe L, Lorini FL, Arcadipane A, et al. Left ventricular systolic function evaluated by strain echocardiography and relationship with mortality in patients with severe sepsis or septic shock: a systematic review and meta-analysis. Crit Care Lond Engl. 2018 Aug 4;22(1):183. 


\section{Tables}

Table 1. Population characteristics $(n=37)$

\begin{tabular}{|c|c|}
\hline Age (years) & $62[53 ; 72]$ \\
\hline Female gender & $17(46 \%)$ \\
\hline Body Mass Index $\left(\mathrm{kg} \cdot \mathrm{m}^{-2}\right)$ & $25[21 ; 28]$ \\
\hline Simplified Acute Physiology Score II & $51[44 ; 64]$ \\
\hline \multicolumn{2}{|l|}{ Preexisting conditions } \\
\hline Chronic arterial hypertension & 19 (51.4\%) \\
\hline Ischemic heart disease & $3(8.1 \%)$ \\
\hline Chronic kidney disease & $6(16.2 \%)$ \\
\hline Diabetes & $12(32.4 \%)$ \\
\hline Obesity & $3(8.1 \%)$ \\
\hline Immunosuppression & $4(10.8 \%)$ \\
\hline Liver cirrhosis & $1(2.7 \%)$ \\
\hline Beta-Blocker & $7(18.9 \%)$ \\
\hline \multicolumn{2}{|l|}{ Source of infection } \\
\hline Lung & $14(37.8 \%)$ \\
\hline Abdomen & $9(24.3 \%)$ \\
\hline Urinary tract & $5(13.5 \%)$ \\
\hline Other & $9(24.4 \%)$ \\
\hline Community-acquired infection & $34(92 \%)$ \\
\hline Norepinephrine at baseline $\left(\mu \mathrm{g} \cdot \mathrm{kg}^{-1} \cdot \mathrm{min}^{-1}\right)$ & $0.8[0.35 ; 1.45]$ \\
\hline Mechanical ventilation at baseline & $23(52.2 \%)$ \\
\hline ICU length of stay (days) & $5[4 ; 11]$ \\
\hline Non-survivors at day 30 & $9(24.3 \%)$ \\
\hline
\end{tabular}

For categorical variables, results are given as number of patients and percentages while for continuous variables as median and interquartile range $\left(25^{\text {th }} ; 75^{\text {th }}\right.$ percentiles $)$. ICU $=$ Intensive Care Unit, $\mathrm{min}=$ minutes. 
Table 2. Hemodynamic and echocardiographic characteristics.

Mean Arterial Pressure (mmHg)

$$
\begin{aligned}
& \text { T1 ( } n=37) \\
& \text { T2 ( } n=24) \\
& \text { T3 ( } n=9)
\end{aligned}
$$

$82[73 ; 90]$

$83[75.75 ; 92.25]$

$81[76 ; 97]$

Systolic Arterial Pressure $(\mathrm{mmHg})^{*}$

$$
\begin{aligned}
& \text { T1 }(n=37) \\
& \text { T2 }(n=24) \\
& \text { T3 }(n=9)
\end{aligned}
$$

$123[104 ; 142]$

$134[119.75 ; 152.25]$

$143[126 ; 145]$

Diastolic Arterial Pressure $(\mathrm{mmHg})$

$$
\begin{aligned}
& \text { T1 ( } n=37) \\
& \text { T2 ( } n=24) \\
& \text { T3 ( } n=9)
\end{aligned}
$$

$61[58 ; 70]$

$63.5[54 ; 74.25]$

$63[53 ; 66]$

Heart rate (beats. $\mathrm{min}^{-1}$ )

$$
\begin{aligned}
& \text { T1 }(n=37) \\
& \text { T2 ( }=24) \\
& \text { T3 }(n=9)
\end{aligned}
$$

$100[84 ; 115]$

$92.5[76 ; 101.25]$

$85[80 ; 96]$

$\operatorname{LVEF}(\%)$

$$
\begin{aligned}
& \text { T1 }(n=33) \\
& \text { T2 ( } n=24) \\
& \text { T3 }(n=9)
\end{aligned}
$$

$$
\begin{gathered}
50[42 ; 60] \\
50[45 ; 60.25] \\
59[50 ; 60]
\end{gathered}
$$

Peak mitral E-wave velocity $\left(\mathrm{m} . \mathrm{s}^{-1}\right)$

$$
\begin{aligned}
& \text { T1 }(n=37) \\
& \text { T2 ( } n=24)
\end{aligned}
$$$$
\text { T3 }(n=9)
$$

$0.8[0.61 ; 0.92]$

$0.8[0.7 ; 0.91]$

$0.8[0.7 ; 1.2]$

Peak mitral A-wave velocity $\left(\mathrm{m} . \mathrm{s}^{-1}\right)$

$$
\begin{aligned}
& \text { T1 }(n=35) \\
& \text { T2 }(n=24) \\
& \text { T3 }(n=8)
\end{aligned}
$$

$$
\begin{gathered}
0.75[0.54 ; 0.98] \\
0.78[0.55 ; 1] \\
0.8[0.69 ; 1]
\end{gathered}
$$

Lateral E/Ea ratio

$$
\begin{aligned}
& \text { T1 }(n=37) \\
& \text { T2 ( } n=24) \\
& \text { T3 (n=9) }
\end{aligned}
$$$$
7.7[6.7 ; 10]
$$$$
8.3[6.8 ; 10.2]
$$$$
8[6.4 ; 10.9]
$$

Peak mitral S-wave velocity $\left(\mathrm{m} . \mathrm{s}^{-1}\right)$

$$
\begin{aligned}
& \text { T1 }(n=34) \\
& \text { T2 }(n=23) \\
& \text { T3 }(n=9)
\end{aligned}
$$

$0.1[0.08 ; 0.12]$

$0.09[0.08 ; 0.12]$

$0.12[0.09 ; 0.16]$

$\operatorname{LVEDV~}(\mathrm{ml})$

$$
\begin{aligned}
& \text { T1 ( } n=34) \\
& \text { T2 ( } n=21) \\
& \text { T3 ( } n=8)
\end{aligned}
$$

Sub Aortic VTI $(\mathrm{cm})^{*}$

$$
\begin{aligned}
& \text { T1 }(n=37) \\
& \text { T2 }(n=24) \\
& \text { T3 }(n=9)
\end{aligned}
$$

$68.5[56.25 ; 81.25]$

$$
75.7[52 ; 96]
$$

$96.85[90 ; 107.67]$

$$
17 \text { [12.45; 21] }
$$

$19.35[15.57 ; 22.65]$

$21[20 ; 25]$ 
TAPSE (mm)
T1 $(n=35)$
$18[15 ; 22]$
T2 ( $n=23)$
$19[16 ; 22.6]$
T3 $(n=9)$
$22[21 ; 26]$

Tricuspid S wave $\left(\mathrm{m} \cdot \mathrm{s}^{-1}\right)$
T1 ( $n=36)$
$0.14[0.11 ; 0.18]$
T2 ( $n=23)$
$0.16[0.12 ; 0.22]$
T3 $(n=9)$
$0.18[0.17 ; 0.21]$

Right Ventricular dilatation
T1 ( $n=37)$
$3(8 \%)$
T2 ( $n=24)$
0
T3 $(n=9)$
0

Results are given as median and interquartile range ( $25^{\text {th }}-75^{\text {th }}$ percentiles) for continuous variables and as number of patients and percentages for categorical variables. For each time and variable, the number of patients for which data were available is specified in brackets ( $n=)$. Ea= tissue doppler mitral annular early diastolic velocity, LVEF = Left Ventricular Ejection Fraction, LVED = Left Ventricular End-Diastolic Volume, TAPSE = Tricuspid Annular Plane Systolic Excursion.

$*: \mathrm{p}<0.05$ between $\mathrm{T} 1$ and $\mathrm{T} 2$ 
Table 3. Biological data

Arterial pH

$$
\begin{aligned}
& \text { T1 }(n=36) \\
& \text { T2 }(n=24) \\
& \text { T3 }(n=9)
\end{aligned}
$$

$\mathrm{P} 02$ ( $\mathrm{mmHg}$ )

T1 $(n=36)$

T2 ( $n=24)$

T3 (n=9)

$\mathrm{PaO} 2 / \mathrm{FiO} 2$ (mmHg)

T1 $(n=21)$

T2 ( $n=24)$

T3 $(n=2)$

$\mathrm{pCO} 2(\mathrm{mmHg})$

$$
\begin{aligned}
& \text { T1 }(n=36) \\
& \text { T2 }(n=24) \\
& \text { T3 (n=9) }
\end{aligned}
$$

Base Excess *

$$
\begin{aligned}
& \text { T1 }(n=26) \\
& \text { T2 }(n=21) \\
& \text { T3 }(n=6)
\end{aligned}
$$

Arterial Lactate $\left(\mathrm{mmol}^{\mathrm{I}} \mathrm{I}^{-1}\right)^{*}$

$$
\begin{aligned}
& \text { T1 }(n=36) \\
& \text { T2 ( } n=24)
\end{aligned}
$$$$
\text { T3 (n=9) }
$$

Troponin (UI)

$$
\begin{aligned}
& \text { T1 }(n=28) \\
& \text { T2 (n=3) } \\
& \text { T3 (n=2) }
\end{aligned}
$$

Bilirubin ( $\left.\mu \mathrm{mol} . \mathrm{I}^{-1}\right)$

$$
\begin{aligned}
& \text { T1 }(n=27) \\
& \text { T2 (n=12) } \\
& \text { T3 (n=7) }
\end{aligned}
$$

Plasma Creatinine ( $\mu \mathrm{mol}^{. \mathrm{I}^{-1}}$ )

$$
\begin{aligned}
& \text { T1 }(n=37) \\
& \text { T2 ( } n=23) \\
& \text { T3 }(n=9)
\end{aligned}
$$

Platelets $\left(. \mathrm{cm}^{-3}\right)$

$$
\begin{aligned}
& \text { T1 ( } n=37) \\
& \text { T2 ( } n=23)
\end{aligned}
$$$$
\text { T3 (n=9) }
$$

$$
\begin{aligned}
& 7.36[7.28 ; 7.41] \\
& 7.40[7.33 ; 7.46] \\
& 7.44[7.35 ; 7.46]
\end{aligned}
$$

$86.45[74.25 ; 127]$

$72.7[66 ; 94.75]$

$77[67.9 ; 83.9]$

$202[152 ; 275]$

$257[186 ; 287]$

$180[173 ; 187]$

$37.95[32.75 ; 43.25]$

$39.5[35.75 ; 46.62]$

$39[35 ; 49]$

$-5[-7.47 ;-1.1]$

$-0.9[-3.4 ; 4.5]$

$1.65[-1.65 ; 7.2]$

$2.7[1.98 ; 3.42]$

$1.6[1.37 ; 2.08]$

$1.3[1.3 ; 2.6]$

$$
42.3[19.17 ; 122.62]
$$

$70.6[68.3 ; 645.8]$

$1731.5[868.75 ; 2594.25]$

$15.9[11.65 ; 23.75]$

$15.85[11.97 ; 54.92]$

$64[16.9 ; 105.4] p$ ?

$133[81 ; 178]$

$153[83.5 ; 217]$

$95[47 ; 144]$

$170[91 ; 329]$

$158[51.5 ; 350.5]$

$112[49 ; 333]$

Results are given as median and interquartile range $\left(25^{\text {th }}-75^{\text {th }}\right.$ percentiles). For each time and variable, the number of patients for which data were available is specified in brackets $(n=)$.

$*: p<0.05$ between T1 and T2 
Table 4. Global Longitudinal Strain for the whole population and according to the vital status at day thirty.

\begin{tabular}{|c|c|c|c|}
\hline View & $\begin{array}{l}\text { Overall } \\
n=37\end{array}$ & $\begin{array}{c}\text { Nonsurvivors (Day 30) } \\
n=9\end{array}$ & $\begin{array}{c}\text { Survivors (Day 30) } \\
n=28\end{array}$ \\
\hline \multicolumn{4}{|c|}{ GLS two-chamber (\%) } \\
\hline$T 1(n=36)$ & $-11.0[-13.25 ;-9.38]$ & $-8.65[-12.25 ;-5.15]$ & $-11.0[-13.25 ;-10.65]$ \\
\hline$T 2(n=23)$ & $-15.0[-15.95 ;-13.05]$ & $-14.0[-19 ;-13.8]$ & $-15.0[-15.88 ;-12.87]$ \\
\hline T3 $(n=9)$ & $-14.0[-15 ;-12]$ & $-14.0[-14.5 ;-12.95]$ & $-14.25[-14.88 ;-12.5]$ \\
\hline \multicolumn{4}{|c|}{ GLS three-chamber (\%) } \\
\hline$T 1(n=36)$ & $-11.0[-16.12 ;-9]$ & $-8.55[-11.55 ;-5.15]$ & $-11.95[-16.52 ;-9.93]$ \\
\hline$T 2(n=23)$ & $-15.4[-19 ;-12.5]$ & $-16.2[-20 ;-15.4]$ & $-14.8[-18.9 ;-12.25]$ \\
\hline$T 2(n=9)$ & $-16.6[-20 ;-15.7]$ & $-19.5[-20.75 ;-15.25]$ & $-16.15[-19.15 ;-15.7]$ \\
\hline \multicolumn{4}{|c|}{ GLS four-chamber (\%) } \\
\hline$T 1(n=36)$ & $-10.8[-13.9 ;-9.47]$ & $-9.35[-10.22 ;-8.6]$ & $-11.8[-16 ;-9.97]$ \\
\hline$T 2(n=23)$ & $-15.0[-18.1 ;-13]$ & $-13.7[-15.5 ;-13]$ & $-15.5[-18.77 ;-13]$ \\
\hline T3 $(n=9)$ & $-15.1[-17 ;-13]$ & $-17[-19.5 ;-13.5]$ & $-15.05[-16 ;-13.5]$ \\
\hline \multicolumn{4}{|l|}{ GLS Average (\%) } \\
\hline $\mathrm{T} 1(\mathrm{n}=35)$ & $-11.0[-14.75 ;-9.55]$ & $-9.1[-10.85 ;-6.6]$ & $-11.0[-15.2 ;-10]$ \\
\hline$T 2(n=23)$ & $-15.6[-17.7 ;-11.55]$ & $-15[-17 ;-14.6]$ & $-15.8[-17.95 ;-11.28]$ \\
\hline T3 $(n=9)$ & $-16.1[-18.3 ;-14.3]$ & $-16.1[-18.05 ;-13.55]$ & $-16.15[-17.98 ;-14.55]$ \\
\hline \multicolumn{4}{|l|}{ RV GLS Average (\%) } \\
\hline$T 1(n=20)$ & $-11.7[-16.95 ;-9.17]$ & $-6.9[-10.7 ;-3.38]$ & $-12.15[-18.5 ;-10.78]$ \\
\hline$T 2(n=16)$ & $-15.8[-20.17 ;-9.6]$ & $-14.6[-19.3 ;-14.3]$ & $-17.0[-19.9 ;-9]$ \\
\hline T3 $(n=6)$ & $-17.2[-22.52 ;-12.17]$ & $-17.05[-19.52 ;-14.57]$ & $-17.55[-23.1 ;-11.55]$ \\
\hline
\end{tabular}

Results are given as median and interquartile range $\left(25^{\text {th }}\right.$ and $75^{\text {th }}$ percentiles). GLS $=$ Global Longitudinal Strain, $\mathrm{RV}$ $=$ Right Ventricle. For each time and variable, the number of patients for which data were available for the whole population is specified in brackets. 
Figures

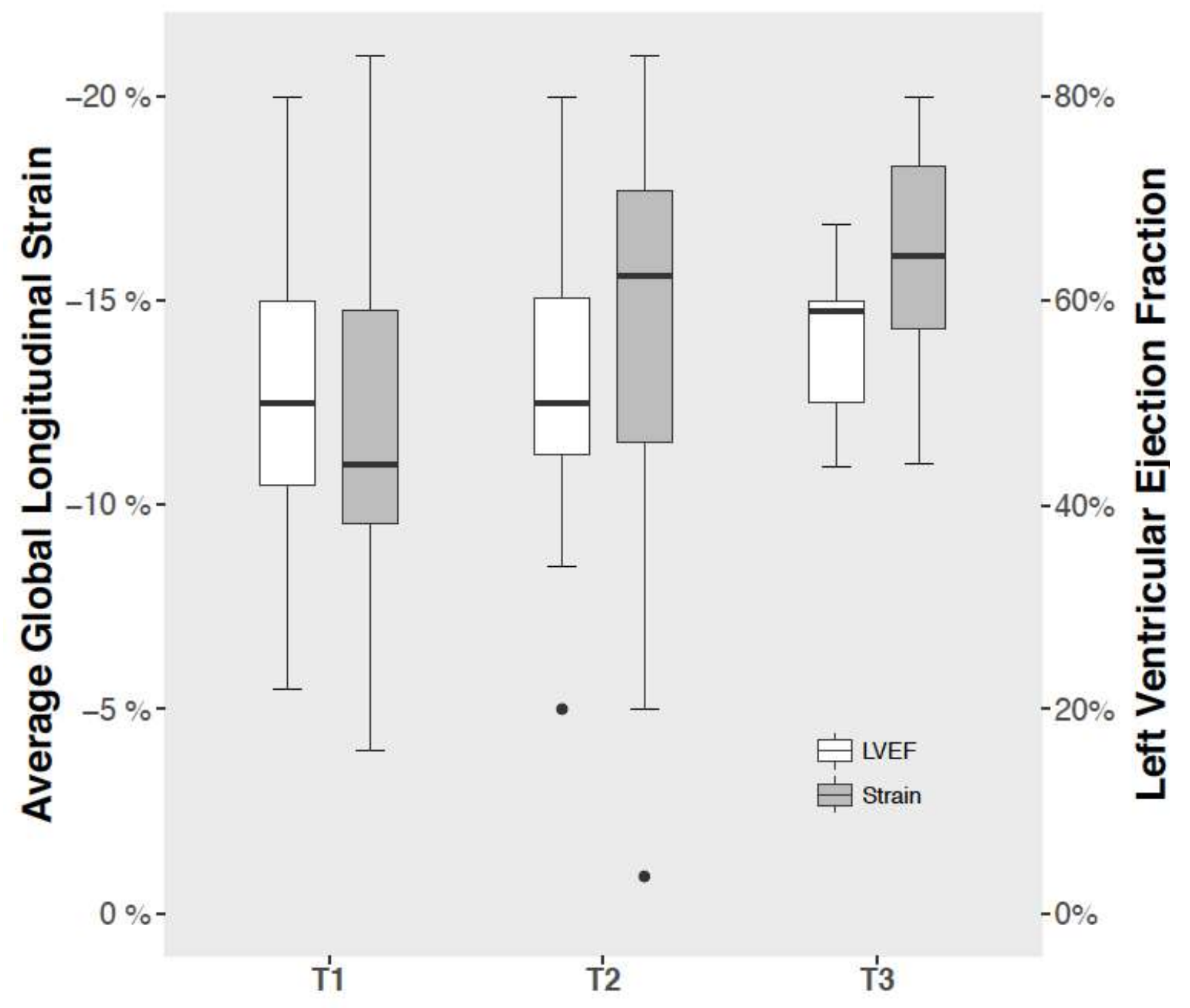

Figure 1. Evolution of longitudinal strain and left ventricle ejection fraction values over time during septic shock (median, interquartile). T1 = day 1, T2 = day 3-5, T3 = day 8 .

lower whisker $=$ smallest observation greater than or equal to lower hinge $-1.5 *$ IQR, upper whisker $=$ largest observation less than or equal to upper hinge $+1.5 *$ IQR). 

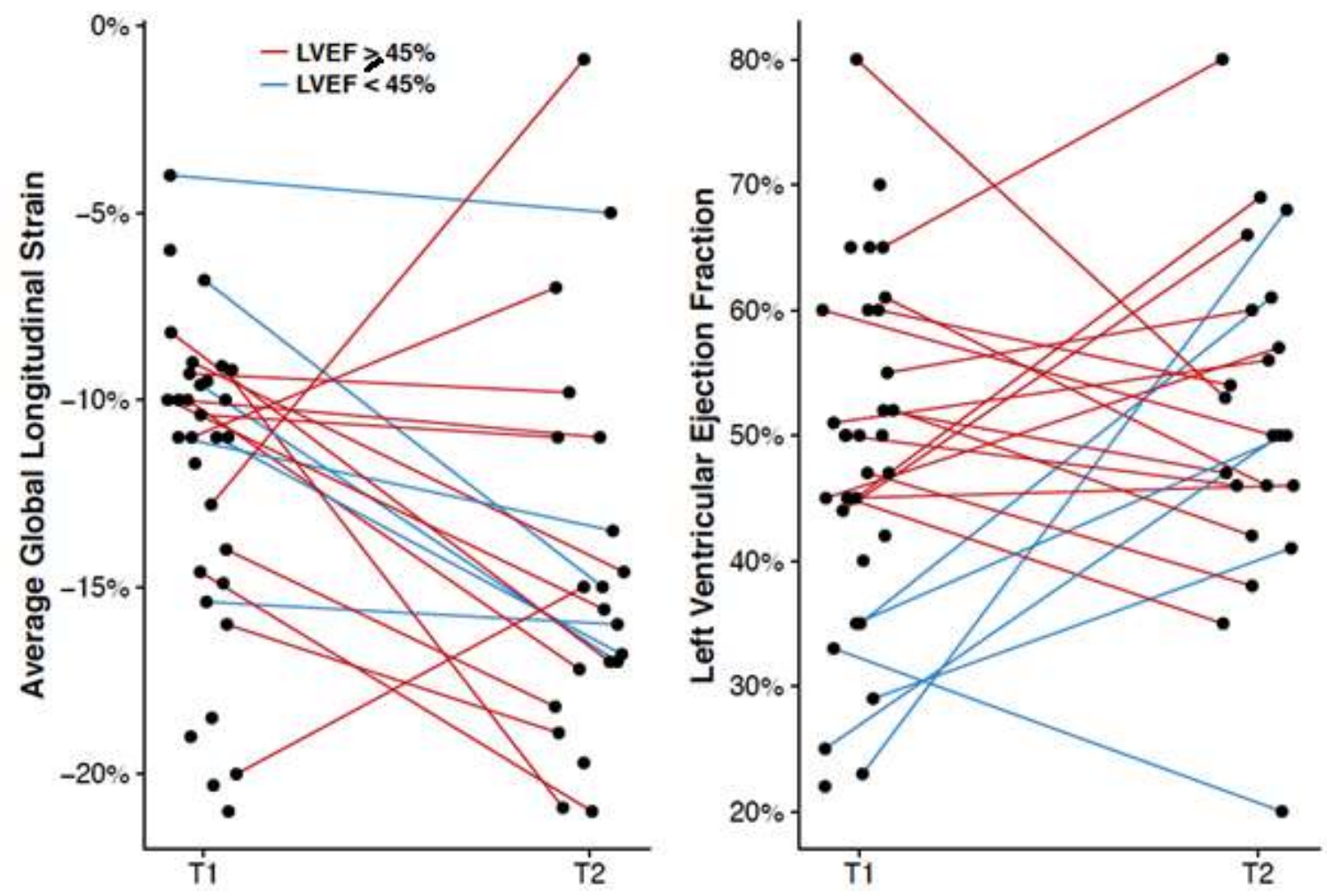

Figure 2. Individual values of global longitudinal strain (Left, 2a) and left ventricle ejection fraction (LVEF) (Right, 2b) between day 1 (T1) and day 3-5 (T2), according to initial LVEF. 


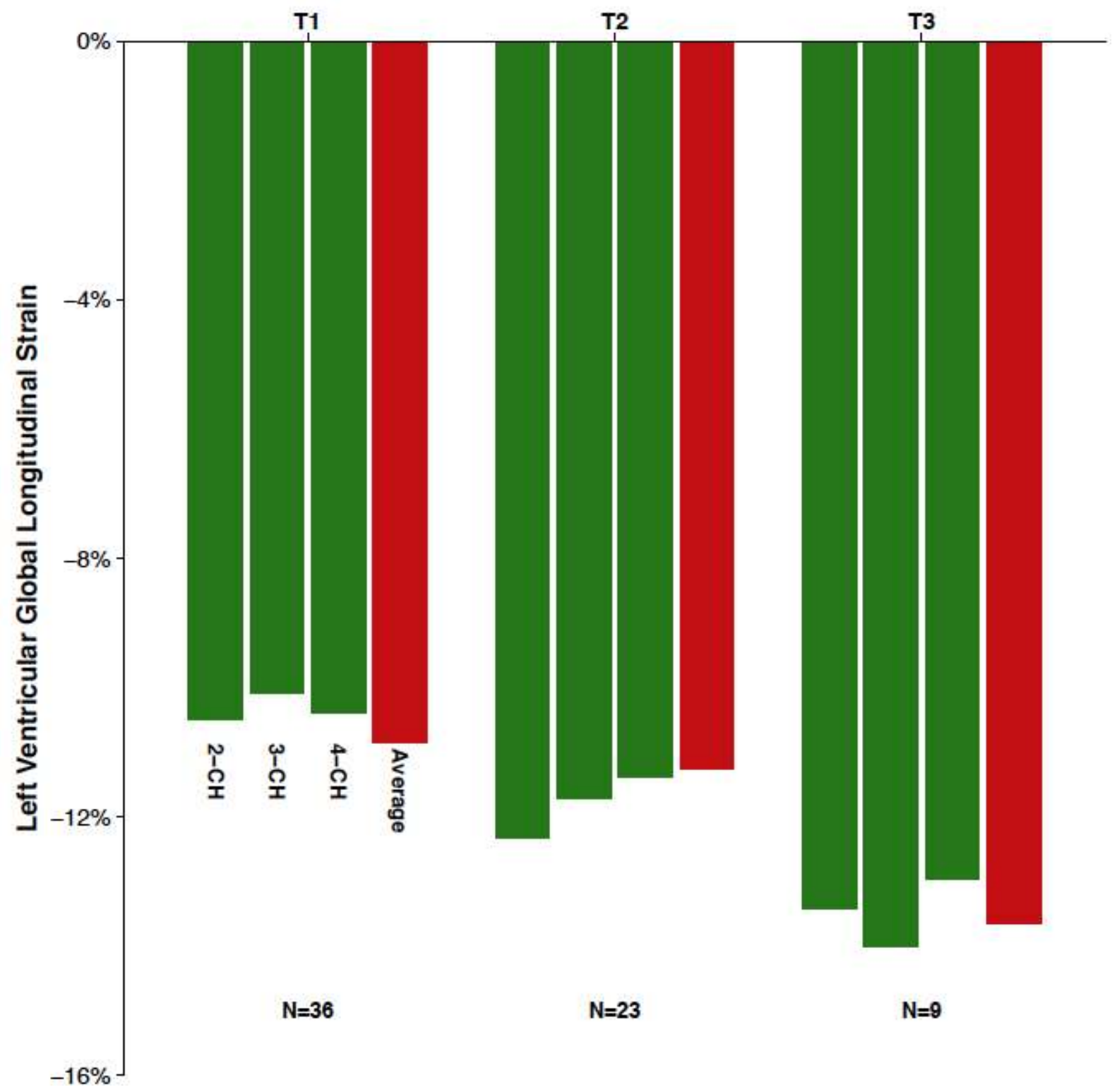

Figure 3. Comparison between mean global longitudinal strain values in 2, 3 and 4 chambers view and the averaged GLS at T1, T2 and T3 exams. T1 = day $1, \mathrm{~T} 2=$ day $3-5, \mathrm{~T} 3=$ day 8. 

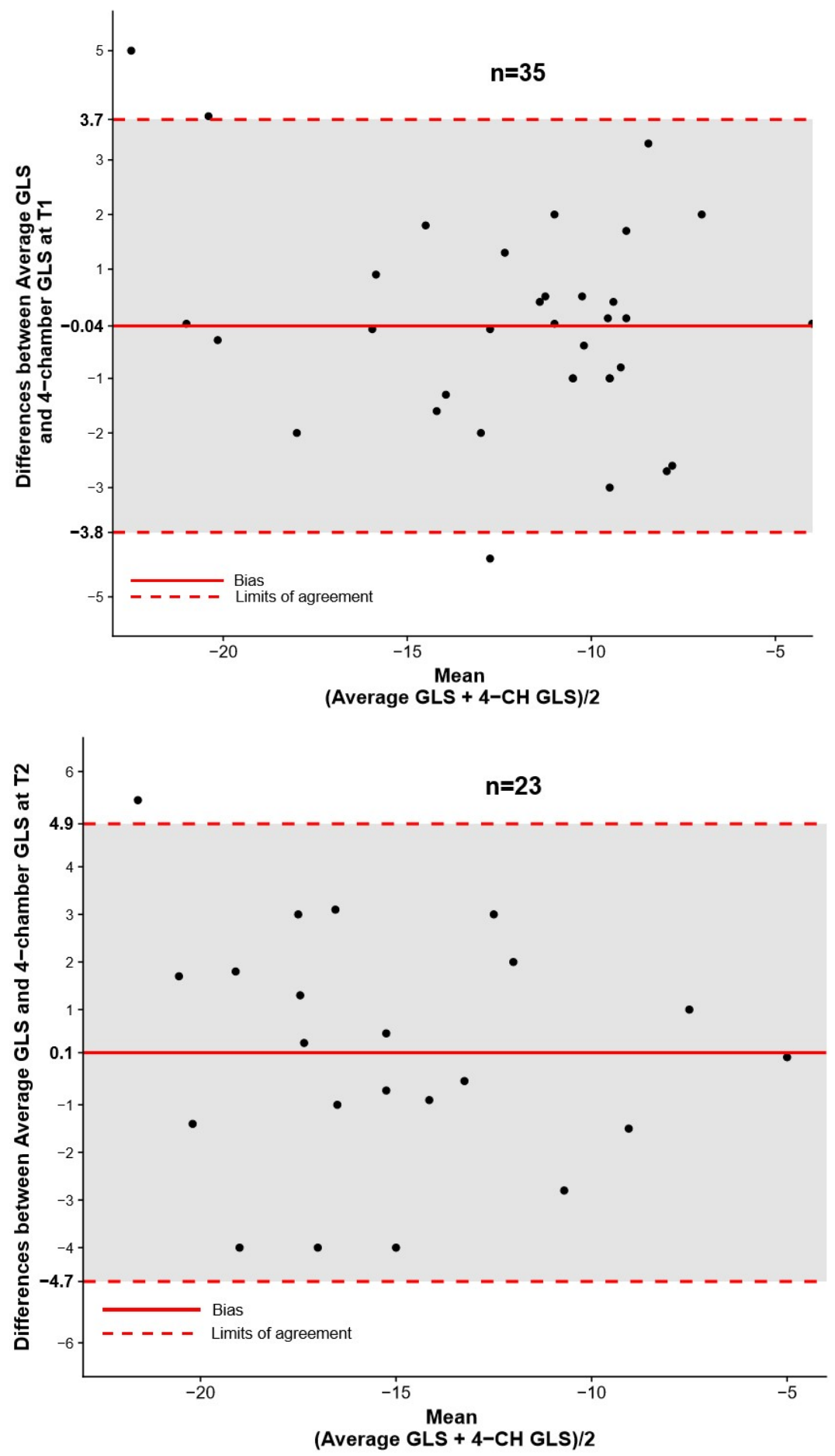

Figure 4. Bland and Altman diagram to assess agreement between average GLS (average of GLS value obtained for 2 chambers, 4 chambers and APLAX views) and four-chamber GLS as a simplified method for strain assessment (single Longitudinal strain: SLS method) in T1 (4a) and $\mathrm{T} 2$ (4b). (computing the mean difference and its $95 \%$ confidence interval and limits of agreement defined as mean difference $\pm 1.96 \mathrm{SD}$ ) 
Annex 1: SLS four chamber

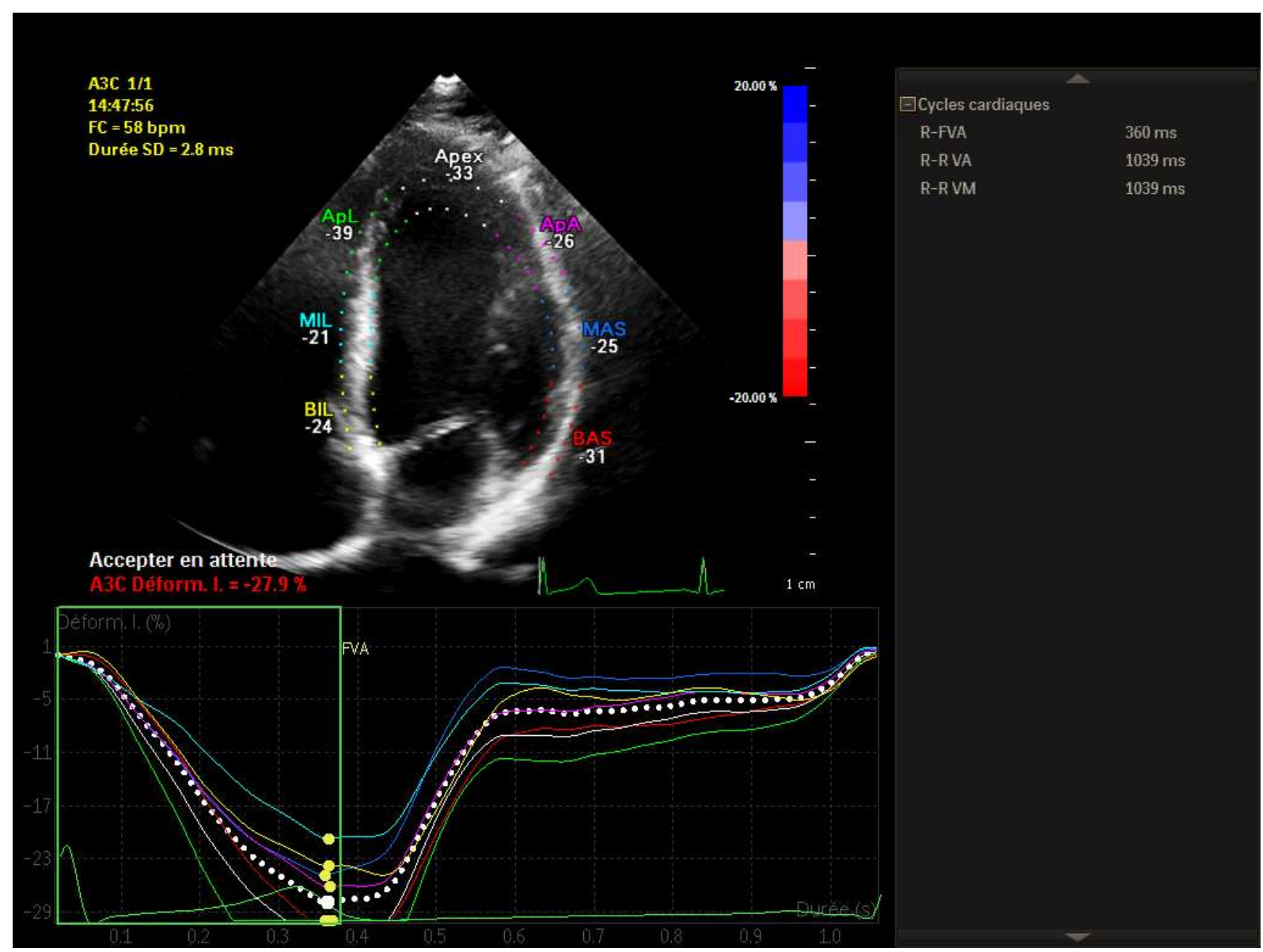




\section{SERMENT}

En présence des Maîtres de cette école, de mes chers condisciples et devant C'effigie d'Hippocrate, je promets et je jure, au nom de l'Etre suprême, d'être fidèle aux lois de ['honneur et de la probité dans l'exercice de la médecine.

$>$ Je donnerai mes soins gratuits à lindigent et n'exigerai jamais un salaire au-dessus de mon travail.

- Admis (e) dans ['intérieur des maisons, mes yeux ne verront pas ce qui s'y passe, ma langue taira les secrets qui me seront confiés, et mon état ne servira pas à corrompre les mours, ni à favoriser le crime.

Respectueux (se) et reconnaissant (e) envers mes Maîtres, je rendrai à leurs enfants ['instruction que j'ai reçue de leurs pères.

Que les hommes m'accordent leur estime si je suis fidèle à mes promesses. Que je sois couvert (e) d'opprobre et méprisé (e) de mes confrères si j'y manque. 


\section{ABSTRACT}

\section{$\underline{\text { Introduction }}$}

GLS is a new tool for systolic function assessment. The present study aims to describe the course of GLS comparing to LVEF during the first days of the septic shock. We also assessed the correlation between GLS values and longitudinal strain measured in apical two three or four chambers. Finally, we assessed the correlation between GLS changes and patient outcome.

\section{Methods}

A prospective observational single center study was performed in the ICU of the Nimes university hospital, France.

After approval of the local ethics committee, all patients admitted to the ICU for septic shock without pre-existing heart disease were eligible

Echocardiography was performed on the first day, and repeated once between day 3 and day 5 then once between day 6 and day 8. LVEF and GLS were acquired in apical two-chamber, fourchamber and long-axis views.

\section{$\underline{\text { Results }}$}

We enrolled 40 consecutive patients. Two patients were excluded for coronary heart disease and one for atrial fibrillation. In all population GLS in T1 is impaired. On T2 exams, a significant improvement of the GLS $(-11,1$ vs $-14,2 p=0,012 ;[0.7 ; 5.5])$ is observed. These variations aren't observed for LVEF which remains stable over time.

We have a good correlation between GLS and longitudinal strain measured on a four chambers view. Evaluated by Bland and Altman method the mean of differences for T1 exams was -0.04 (0.7: 0.62).

\section{$\underline{\text { Conclusion }}$}

Myocardial strain is depressed at the early phase of septic shock and improves over time. A single measurement of GLS in a 4 chambers view appears sufficient at bedside as compared to complete myocardial evaluation (2, 4 chambers and APLX views). Finally, our results support the idea that GLS at day 1 (and not subsequent days) is associated with patient outcome.

Key words: speckle-tracking, strain, Septic cardiomyopathy, echocardiography, LVEF, systolic, septic shock, four chambers. 